\title{
Demagnetization via Nucleation of the Nonequilibrium Metastable Phase in a Model of Disorder
}

\author{
Pablo I. Hurtado, 1,2 , * J. Marro ${ }^{2}$, and P.L. Garrido ${ }^{2}$ \\ ${ }^{1}$ Laboratoire des Colloïdes, Verres et Nanomatériaux, Université Montpellier II, Montpellier 34095, France \\ ${ }^{2}$ Instituto "Carlos I" de Física Teórica y Computacional, and Departamento de Electromagnetismo y Física de la Materia, \\ Universidad de Granada, E-18071 Granada, España
}

\begin{abstract}
We study both analytically and numerically demagnetization via nucleation of the metastable phase in a two-dimensional nonequilibrium Ising ferromagnet at temperature $T$. Canonical equilibrium is dynamically impeded by a weak random perturbation which models homogeneous disorder of undetermined source. We present a simple theoretical description, in perfect agreement with Monte Carlo simulations, assuming that the decay of the nonequilibrium metastable state is due, as in equilibrium, to the competition between the surface and the bulk. This suggests one to accept a nonequilibrium free-energy at a mesoscopic/cluster level, and it ensues a nonequilibrium surface tension with some peculiar low $-T$ behavior. We illustrate the occurrence of intriguing nonequilibrium phenomena, including: $(i)$ stochastic resonance phenomena at low $T$ which stabilize the metastable state as temperature increases; (ii) reentrance of the limit of metastability under strong nonequilibrium conditions; and (iii) noise-enhanced propagation of domain walls. The cooperative behavior of our system, which is associated to the interplay between thermal and nonequilibrium fluctuations, may also be understood in terms of a Langevin equation with additive and multiplicative noises. We also studied metastability in the case of open boundaries as it may correspond to a magnetic nanoparticle. We then observe the most irregular relaxation triggered by the additional surface randomness. In particular, at low $T$, the relaxation becomes discontinuous as occurring by way of scale-free avalanches, so that it resembles the type of relaxation reported for many complex systems. We show that this results from the superposition of many demagnetization events, each with a well-defined scale which is determined by the curvature of the domain wall at which it originates. This is an example of (apparent) scale invariance in a nonequilibrium setting which is not to be associated with any familiar kind of criticality.
\end{abstract}

KEY WORDS: metastable state, nonequilibrium, kinetic Ising model, nucleation, critical droplet, stochastic resonance, surface effects, disorder, scale-invariant noise, avalanches.

\section{INTRODUCTION}

Many different natural phenomena involve metastable states that, eventually, decay via nucleation. Some familiar examples appear in the flow of electrical current through resistors, relaxation in amorphous materials, glasses and gels, domain wall motion in hysteretic disordered magnets, granular media evolution, earthquake dynamics, protein conformations, or false vacuum states in quantum field theory. There is a great amount of information concerning these situations but a full microscopic theory of metastability and nucleation is elusive $(1 ; ; 2 ; 3 ;$; $;$; ; 6 ; 7 ; 8 ; 9 ). To begin with, there are two main coupled difficulties. One is that metastability concerns dynamics $(\underline{3} ; 4)$. The systems of interest typically show a complex free-energy landscape with (many) local minima, which are metastable in the sense that they trap the system for a long time. One may imagine that, eventually, relaxation occurs when the system after long wandering finds a proper path between the minima. This results in a complicate coupling of dynamics and thermodynamics (10). A second difficulty is that many systems of interest cannot reach thermal equilibrium even after relaxation. In general, they are open to the environment, which often induces currents of matter or energy, or they are subject to agents which impose opposing tendencies which typically break detailed balance (11). These perturbations result in a final steady state which cannot be described by a Gibbsian measure, i.e. a nonequilibrium stationary state. Consequently, thermodynamics and ensemble statistical mechanics do not hold in these systems, which is a serious drawback.

These difficulties make the field most suitable for simple-model analysis. Indeed, the (two-dimensional) kinetic Ising model has been the subject of many studies of metastability not only in the case of periodic boundaries $(6 ;$; $12 ; 13 ; 14)$ but also for finite lattices with free boundaries $(15 ; 16 ; 17 ; 18)$. The latter try to capture some of the physics of demagnetization in very dense media where magnetic particle sizes typically range from mesoscopic down to atomic levels. If one keeps oneself away from specific models, metastable states are often treated in the literature as rare equilibrium states, at least for times much shorter that the relaxation time, and it has been shown that one may define

*Electronic address: phurtado@onsager.ugr.es 
a metastable state in a properly constrained (equilibrium) ensemble (2; 3 ; 9 ), and that most equilibrium concepts may easily be adapted $(4 ; 5 ; 6 ; 6 ; 8)$.

In this paper we present a detailed study of metastability (and nucleation) in a nonequilibrium model. In order to deal with a simple microscopic model of metastability, we study a two-dimensional kinetic Ising system, as in previous studies. However, for the system to exhibit nonequilibrium behavior, time evolution is defined here as a superposition of the familiar thermal process at temperature $T$ and a weak completely-random process. This competition is probably one of the simplest, both conceptually and operationally, ways of impeding equilibrium. Furthermore, one may argue that it captures some underlying disorder induced by random impurities or other causes which are unavoidable in actual samples. The specific origin for such dynamic randomness will vary with the situation considered. We mention that a similar mechanism has already been used to model the macroscopic consequences of rapidly-diffusing local defects (11) and quantum tunneling (16) in magnetic materials, for instance.

The weakest perturbation of that kind happens to modify essentially the canonical equilibrium. We observe, however, some structural similarity at the mesoscopic level which suggests that cluster dynamics is, as in equilibrium, the consequence of a competition between the surface and the bulk. Therefore, we postulate the existence of a nonequilibrium free-energy and an associated nonequilibrium surface tension. These functions are formally similar to the ones in equilibrium but behave peculiarly due to the different nature of the system states. In fact, two main predictions follow that are counter intuitive. One is that, at low $T$, the stability of the metastable state is enhanced by increasing temperature, as a consequence of resonance between thermal and nonequilibrium noises. The second main prediction is that the limit of metastability or pseudospinodal, which separates the metastable phase from the unstable one, exhibits reentrant behavior as a function of $T$ for strong enough nonequilibrium conditions. Our predictions are nicely confirmed in related Monte Carlo (MC) simulations. We also show that the most important aspects of the model behavior are contained in a Langevin-like description which contains a multiplicative noise in addition to the more familiar additive one.

The theory above concerns an infinite system, and the related computer simulations are, therefore, for lattices with periodic boundary conditions. However, we also studied other types of boundary conditions, including free borders. Previous work showed that free borders, which are most relevant when dealing with magnetic nanoparticles, for instance, determine importantly the particle demagnetization $(17 ; 18)$. We here confirm this and observe that, for free borders, but not for periodic boundary conditions, demagnetization occurs, via avalanches, through many different metastable-like configurations. These avalanches exhibit power-law size and lifetime distributions in a way that closely resembles the relaxation in many cases in nature. We show that this is a consequence in the model of the superposition of different events, each with a typical scale which is determined by the curvature of the interface at which the avalanche originates.

The paper is organized as follows. We define the model in the next section. In section III we set the basis of our approach. In particular, $\amalg$ III.A concerns a mean-field approximation which allows for a discussion in $₫$ III.B of the nonequilibrium surface tension. The next subsection introduces our ansatz for a nonequilibrium free-energy cost of excitations in the metastable phase, and we test the ensuing predictions against MC simulations. In $₫ \amalg I I$ we present a Langevin theory which is also a good description of the nonequilibrium situation of interest here. \$III.E is devoted to study the limit of metastability. In section IV we study the case of open boundaries. The statistics of the resulting avalanches are analyzed in $₫ \mathrm{IV.A}$, and $₫ \mathrm{IV.B}$ and $\oint \mathrm{IV.C}$ are devoted to discussions, respectively, of our observations and of its possible extension to interpret avalanche data from actual systems. Finally, section $\$ \nabla$ contains a brief conclusion. Some technical details are left for the appendices.

\section{THE MODEL}

Consider a two-dimensional square lattice of side $L$ and, for the moment, periodic boundary conditions (or, eventually, the thermodynamic limit condition $L \rightarrow \infty$ ). We define a spin variable $s_{i}= \pm 1$ at each node, $i \in\left[1, N \equiv L^{2}\right]$. Spins interact among them, and with an external magnetic field $h$, via the Ising Hamiltonian function

$$
\mathcal{H}(\mathbf{s})=-\sum_{|i-j|=1} s_{i} s_{j}-h \sum_{i=1}^{N} s_{i},
$$

where $\mathbf{s}=\left\{s_{i}\right\}$ and the first sum runs over all nearest-neighbors (NN) pairs. Time evolution proceeds by stochastic dynamics consisting of single-spin flips with the transition rate

$$
\omega\left(\mathbf{s} \rightarrow \mathbf{s}^{i}\right)=p+(1-p) \Psi\left(\beta \Delta \mathcal{H}_{i}\right),
$$

where $0<p<1$ is a parameter, $\mathbf{s}$ and $\mathbf{s}^{i}$ stand, respectively, for the system configuration before and after flipping the spin at node $i, \Delta \mathcal{H}_{i}$ is the energy cost of the flip, and $\beta=1 / T$. The (arbitrary) function in (2) is set $\Psi(x)=$ 


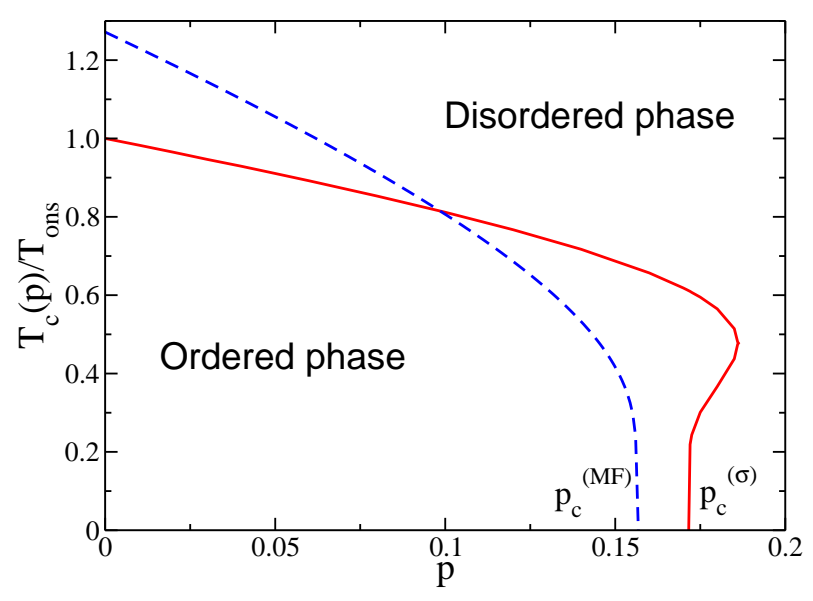

FIG. 1 (Color) Phase diagram for the nonequilibrium Ising ferromagnet of interest here. The solid (red) line corresponds to the critical temperature as obtained from the nonequilibrium surface tension in $\coprod$ The order disappears at $T=0$ for $p>p_{c}=(\sqrt{2}-1)^{2} \approx 0.17$. Notice, however, the reentrant blob for $p_{c}<p<p_{c}^{*} \approx 0.186$ and intermediate temperature. The dashed (blue) line is derived from a first-order (pair approximation) mean-field theory, namely, eq. (8) in $₫$ III.A.

$\mathrm{e}^{-x}\left(1+\mathrm{e}^{-x}\right)^{-1}$, corresponding to the so-called Glauber rate, in our simulations. However, we shall also refer below to the case $\Psi(x)=\min \left[1, \mathrm{e}^{-x}\right]$ which corresponds to the Metropolis rate.

For any $p$, two different heat baths compete in (2): One is at temperature $T$ and operates with probability $(1-p)$, while the other induces completely-random (infinite temperature) spin flips with probability $p$. As a consequence of this competition, a nonequilibrium steady state sets in asymptotically which cannot be characterized by a Gibbsian invariant measure $(11 ; 19)$. In particular, the rate (2) violates detailed balance for any $p>0$. However, the system reduces for $p=0$ to the familiar Ising model with rate $\Psi$ which goes asymptotically to the equilibrium state for temperature $T$ and Hamiltonian $\mathcal{H}$. This model is a particular case of a more general class of systems, characterized by a coupling to a number of different heat baths leading to nonequilibrium behavior, whose static properties have received considerable attention in recent years (11).

For zero magnetic field, the model exhibits an order-disorder continuous phase transition at critical temperature $T_{c}(p)<T_{c}(p=0) \equiv T_{\text {ons }}$, where $T_{\text {ons }}=2 / \ln (1+\sqrt{2})$ is the Onsager temperature. Fig. 1 shows the model phase diagram $T_{c}(p)$ as obtained by two different approaches, as described in $\amalg$ II.A and $\amalg$ III.B respectively. In the former case, corresponding to a first-order (pair) mean-field approximation, the predicted equilibrium $(p=$ 0 ) critical temperature is the Bethe temperature $T_{\text {bethe }}=4 / \ln 4 \approx 1.27 T_{\text {ons }}$, and order disappears at $T=0$ for $p>p_{c}^{(M F)}=5 / 32 \approx 0.156$. A more accurate estimation follows from the nonequilibrium surface tension $\sigma_{0}$ in III.B. In this case, one recovers the correct equilibrium limit, and order is predicted to disappear at $T=0$ for $p>p_{c}^{\left(\sigma_{0}\right)}=(\sqrt{2}-1)^{2} \approx 0.1716$ (in agreement with MC results $(11)$ ). However, the anomalous behavior of $\sigma_{0}$ that we describe in $₫ \underline{I I I . B}$ implies the emergence of an intermediate $-T$ region for $p_{c}<p<p_{c}^{*} \approx 0.18625$ where order sets in. This reentrant behavior of $T_{c}(p)$, which is shown in Fig. 1, is reminiscent of the one in the phase diagram for systems subject to multiplicative noise, as discussed below.

\section{NONEQUILIBRIUM METASTABILITY AND NUCLEATION}

In order to produce metastability in the above nonequilibrium setting, we initialize the system in a state with all spins up, $s_{i}=+1, i=1 \ldots N$. For small $h<0$ and $T<T_{c}(p)$, this configuration quickly relaxes to a metastable state with magnetization $m=N^{-1} \sum_{i=1}^{N} s_{i}>0$. There is, however, a tendency of the spins to line up in the direction of the field, which competes with the tendency to maintain the local order induced by the spins mutual interactions. The result for small $|h|$ is a metastable state of long lifetime, i.e., the system fluctuates around the metastable minimum for a long time, though eventually decays toward the truly stable phase, which has $m<0$.

\section{A. The Metastable Phase: Mean-Field Theory (Pair Approximation)}

The resulting metastable state is homogeneous at mesoscopic scales for times shorter than its relaxation time, as expected (2; 3), and we can exploit this feature. In particular, we shall assume that: $(i)$ the system is completely 


\begin{tabular}{|c||c|c|c|}
\hline Class & Central spin & Number of up neighbors & $\Delta \mathcal{H}$ \\
\hline \hline 1 & +1 & 4 & $8+2 \mathrm{~h}$ \\
\hline 2 & +1 & 3 & $4+2 \mathrm{~h}$ \\
\hline 3 & +1 & 2 & $2 \mathrm{~h}$ \\
\hline 4 & +1 & 1 & $-4+2 \mathrm{~h}$ \\
\hline 5 & +1 & 0 & $-8+2 \mathrm{~h}$ \\
\hline \hline 6 & -1 & 4 & $-8-2 \mathrm{~h}$ \\
\hline 7 & -1 & 3 & $-4-2 \mathrm{~h}$ \\
\hline 8 & -1 & 2 & $-2 \mathrm{~h}$ \\
\hline 9 & -1 & 1 & $4-2 \mathrm{~h}$ \\
\hline 10 & -1 & 0 & $8-2 \mathrm{~h}$ \\
\hline \hline
\end{tabular}

TABLE I Spin classes for the two-dimensional Ising model with periodic boundary conditions. The last column shows the energy cost of flipping the central spin at each class.

homogeneous, and (ii) it can be described by the average density of up spins, $\rho(+)$, and by NN (pair) correlations as captured by the density $\rho\left(s, s^{\prime}\right)$ of NN spins. Under these assumptions, all the spins will behave in the same manner, and the evolution of a spin will depend exclusively on its current state and on its four NNs. Denoting $x \equiv \rho(+)$ and $z \equiv \rho(+,+)$, we may write $\rho(-)=1-x, \rho(+,-)=\rho(+)-\rho(+,+)=x-z$ and $\rho(-,-)=1+z-2 x$. In this approximation, the probability $Q(s, n)$ of finding a spin in a state $s= \pm 1$ surrounded by $n \in[0,4]$ up NN spins is

$$
Q(s, n)=\left(\begin{array}{l}
4 \\
n
\end{array}\right) \rho(s) \rho(+\mid s)^{n} \rho(-\mid s)^{4-n}=\left(\begin{array}{l}
4 \\
n
\end{array}\right) \frac{\rho(+, s)^{n} \rho(-, s)^{4-n}}{\rho(s)^{3}},
$$

where $\rho( \pm \mid s)=\rho( \pm, s) / \rho(s)$ are marginal densities. The pair of indexes $(s, n)$ defines the spin class to which the given spin belongs to. For periodic boundary conditions, there are 10 different spin classes, as shown in Table I. The cost $\Delta \mathcal{H}(s, n)$ of flipping any spin within a class is the same, so that the transition rate $\omega\left(\mathbf{s} \rightarrow \mathbf{s}^{i}\right) \equiv \omega(s, n)$ in (2) only depends on $s$ and $n$, the spin at a node $i$ and the number of its up NN neighbors, respectively.

This information may be used to write down rate equations for the two relevant observables in our mean field approximation, namely $x$ and $z$. In particular, the change in $x$ when a spin $s$ with $n$ up neighbors flips is $\delta x(s, n)=-s$, whereas the change in $z$ in the same process is $\delta z(s, n)=-s n / 4$. We thus obtain $(11 ; 22)$ :

$$
\begin{aligned}
& \frac{\mathrm{d} x}{\mathrm{~d} t}=G_{1}(x, z) \equiv \sum_{n=0}^{4} G(x, z ; n), \\
& \frac{\mathrm{d} z}{\mathrm{~d} t}=G_{2}(x, z) \equiv \sum_{n=0}^{4} \frac{n}{4} G(x, z ; n),
\end{aligned}
$$

where

$$
G(x, z ; n) \equiv\left(\begin{array}{l}
4 \\
n
\end{array}\right)\left[\frac{(x-z)^{n}(1+z-2 x)^{4-n}}{(1-x)^{3}} \omega(-, n)-\frac{z^{n}(x-z)^{4-n}}{x^{3}} \omega(+, n)\right]
$$

Eqs. (4)-(6) correspond to a mean-field Pair Approximation (11; 23). This is a dynamic generalization of the firstorder cluster variation method by Kikuchi (24), and has been used to study other nonequilibrium systems with success (23). Our interest here is on the stationary solutions, $G_{1}\left(x_{\mathrm{st}}, z_{\mathrm{st}}\right)=G_{2}\left(x_{\mathrm{st}}, z_{\mathrm{st}}\right)=0$. Both stable and metastable solutions are locally stable against small perturbations, which requires the associated Lyapunov exponents to be negative. This yields the necessary and sufficient conditions

$$
\begin{gathered}
\left(\partial_{x} G_{1}\right)_{\mathrm{st}}+\left(\partial_{z} G_{2}\right)_{\mathrm{st}}<0 \\
\left(\partial_{x} G_{1}\right)_{\mathrm{st}}\left(\partial_{z} G_{2}\right)_{\mathrm{st}}-\left(\partial_{z} G_{1}\right)_{\mathrm{st}}\left(\partial_{x} G_{2}\right)_{\mathrm{st}}>0 .
\end{gathered}
$$

On the other hand, the condition $\left(\partial_{x} G_{1}\right)_{\text {st }}=0$ signals for $h=0$ an incipient or marginal instability corresponding to an order-disorder phase transition with critical point $\left(x_{\mathrm{st}}^{\mathrm{c}}, z_{\mathrm{st}}^{\mathrm{c}}\right)=(1 / 2,1 / 3)$. It follows that

$$
T_{c}(p)=-4\left[\ln \left(\frac{3}{4} \sqrt{\frac{1-4 p}{1-p}}-\frac{1}{2}\right)\right]^{-1} \text {. }
$$




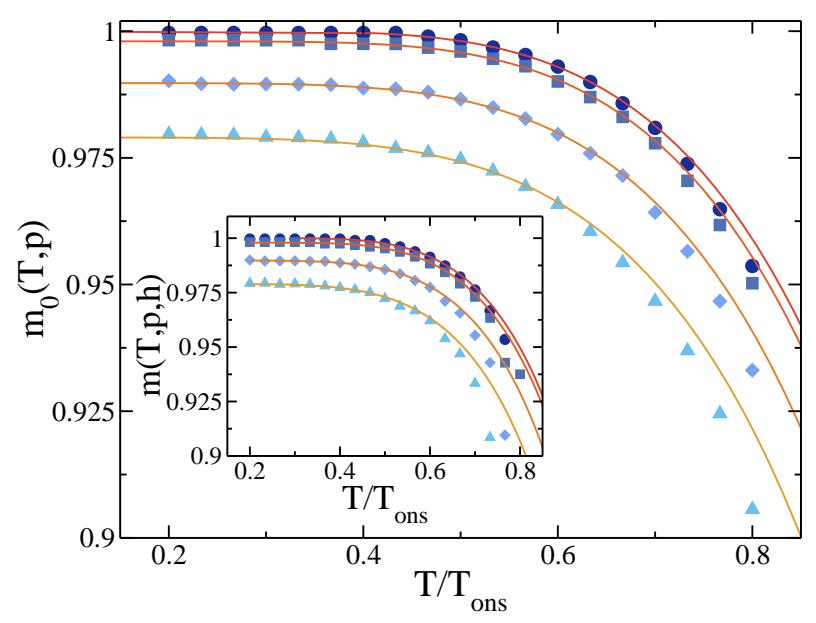

FIG. 2 (Color) Temperature dependence of the steady-state magnetization for $h=0$ and, from top to bottom, $p=0,0.001$, 0.005 , and 0.01. Lines are results from the mean-field (pair) approximation. Inset: Magnetization in the metastable phase vs. $T$ for $h=-0.1$ and the same same values of $p$ as in the main graph.

This function is depicted in Fig. 1 (see the related discussion above in $\left\{(\mathrm{II})\right.$. The stationary state $\left(x_{\mathrm{st}}, z_{\mathrm{st}}\right)$ may be obtained numerically from the nonlinear equations $G_{1}\left(x_{\mathrm{st}}, z_{\mathrm{st}}\right)=G_{2}\left(x_{\mathrm{st}}, z_{\mathrm{st}}\right)=0$ subject to the local stability conditions in eq. (7). For $h=0$, the up-down symmetry leads to pairs of locally-stable steady solutions, namely $\left(x_{\mathrm{st}}, z_{\mathrm{st}}\right)$ and $\left(1-x_{\mathrm{st}}, 1+z_{\mathrm{st}}-2 x_{\mathrm{st}}\right)$. The result is illustrated in Fig. 2. This also illustrates the expected agreement with Monte Carlo results at low and intermediate temperature for any $p$. In particular, this agreement is good for temperatures below $0.75 T_{c}(p)$. The fact that increasing $p$ at fixed $T$ decreases the magnetization reveals that the nonequilibrium perturbation tends to increase disorder, as expected. In this way we can identify the parameter $p$, which weights the competition between different heat baths leading to nonequilibrium behavior, as a source of nonequilibrium noise, as compared with thermal fluctuations induced by $T$. For small enough fields, the situation closely resembles the case $h=0$, but the up-down symmetry is now broken, and locally-stable steady states with magnetization opposite to the applied field are metastable, see inset to Fig. 2.

\section{B. Nonequilibrium Surface Tension}

The above mean-field approximation neglects fluctuations, so that it cannot account for the relaxation of the metastable phase. One expects that, for long enough times, fluctuations will allow for a decay toward the stable phase. Direct inspection of escape configurations as the ones in Fig. 3 indicates that the metastable-stable transition is a highly inhomogeneous process triggered by large stable-phase clusters, and a detailed comparison (not shown) suggests that the nonequilibrium and equilibrium cases are characterized by the same type of relevant excitations. These grow or shrink into the metastable sea depending upon the competition between their surface, which hampers cluster growth, and their bulk, which favors it.

The nucleation and growth of a cluster is controlled in equilibrium, $p=0$, by the zero-field interfacial free-energy, or surface tension. For $p>0$, even though a proper bulk free-energy function does not exist, it seems sensible to assume that the properties of the nonequilibrium interface will be determined by a sort of nonequilibrium or effective surface tension $\sigma_{0}(T, p)^{1}$. This relies on the assumption that the normalization of the probability measure for interface configurations can be interpreted as a nonequilibrium partition function. More formally, let $\mathbf{y}$ denote an interface microscopic configuration - e.g., the set of heights $y_{k}, k=1 \ldots L$, that characterize the steps of the interface , and $Z^{-1} \mu(\mathbf{y} ; T, p)$ the associated invariant measure, which for $0<p<1$ is non-Gibbsian. Then, we define $\sigma_{0}(T, p)=-(\beta L)^{-1} \ln Z(25)$. Similar hypotheses have already been tested in relation with other nonequilibrium phenomena. For instance, concerning nonequilibrium phase transitions, the distribution of complex zeros of the invariant measure normalization factor has recently been shown to obey the Lee-Yang picture in the case of the asymmetric simple exclusion process (20) and for systems with absorbing states (21); see also (22; 25). Interestingly,

\footnotetext{
${ }^{1}$ In general, the surface tension depends on the orientation angle $\phi$ of the interface with respect to one of the lattice axes, $\sigma_{0}(\phi, T, p)$. However, the surface tension entering nucleation theory is the one defined along a primitive lattice vector $(\underline{4} ;$; $;$; 6 ; 7 ), so $\phi=0$ henceforth.
} 


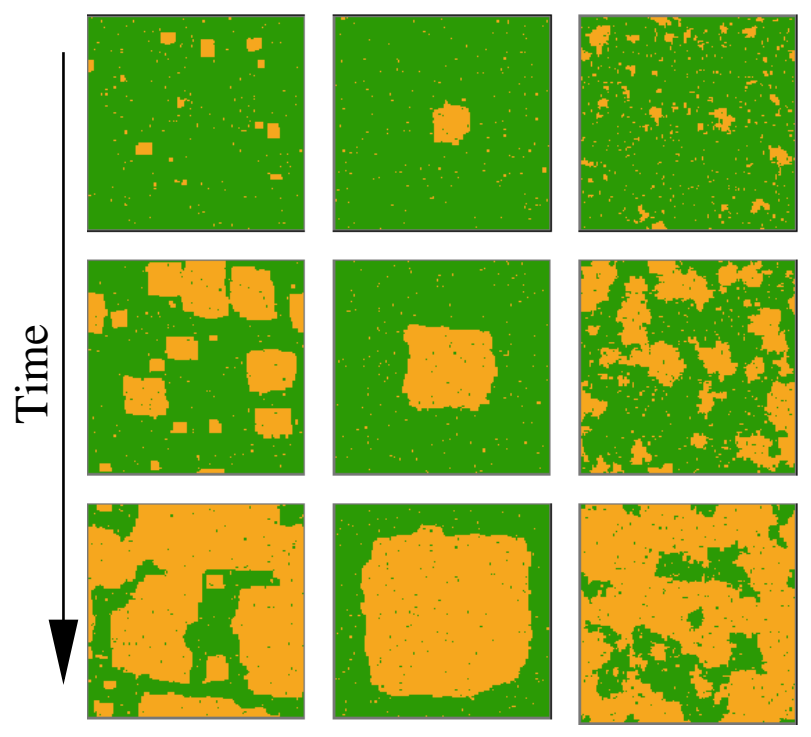

FIG. 3 (Color) Configurations as the system escapes from the metastable state, at different times as indicated, for $L=128$, $p=0.01, h=-0.25$, and, from left to right, $T=0.1,0.3$ and 0.7 in units of $T_{\text {ons }}$.

the normalization $Z$ is defined in general up to a multiplicative factor (20). This is related to the fact that one has to arbitrarily choose a reference interface configuration from which to measure the relative probabilities of all other microscopic configurations. However, in the case of our model, the ambiguity in $Z$ can be easily resolved by noting that a well-defined equilibrium limit exists, $p=0$. In this way, demanding $Z$ to converge to the right equilibrium limit for $p=0$, the ambiguity disappears. In more general nonequilibrium cases, where no proper equilibrium limit can be defined, it is expected that the spurious multiplicative factor won't play any relevant physical role (20).

Computing analytically the exact interfacial invariant measure $Z^{-1} \mu$ is beyond our goals, but a solid-on-solid approximation (26) will suffice. It thus follows (we refer the reader to Ref. (25) for further details) that $\sigma_{0}(T, p)$ has non-monotonous $T$ dependence for any $p>0$ and a maximum at a non-trivial value of $T$ which depends on $p$, see Fig. 4. This peculiar behavior can be understood in simple terms. From low- and high- $T$ expansions of $\sigma_{0}(T, p)$, one obtains that

$$
\sigma_{0} \approx \frac{T}{T_{\mathrm{ef}}^{(\mathrm{I})}} \sigma_{\mathrm{e}}+\text { small corrections }
$$

where $T_{\mathrm{ef}}^{(\mathrm{I})}(T, p)$ is an effective interfacial temperature and $\sigma_{\mathrm{e}}(T)$ is the exact equilibrium surface tension $(27)$. At high- $T$, where thermal fluctuations dominate over the nonequilibrium perturbation, $T_{\text {ef }}^{(\mathrm{I})}$ is proportional to $T$ - see inset to Fig. 4-, so that $\sigma_{0}$ is proportional to $\sigma_{\mathrm{e}}$ which decreases with $T$. However, $T_{\mathrm{ef}}^{(\mathrm{I})}$ saturates to a constant as $T \rightarrow 0$, namely, $\lim _{T \rightarrow 0} T_{\mathrm{ef}}^{(\mathrm{I})}(T, p)=2 / \ln [(1-\sqrt{p}) /(p+\sqrt{p})](25)$. This, which is due to non-vanishing nonequilibrium fluctuations, implies that $\sigma_{0}$ is a linearly-increasing function of $T$ at low- $T$. Consequently, it follows a non-monotonous $T$-dependence of $\sigma_{0}$ with a maximum which roughly agrees with the crossover observed in $T_{\text {ef }}^{(\mathrm{I})}$.

The properties of the nonequilibrium interface may be obtained from the resulting $\sigma_{0}(T, p)$. For instance, the temperature dependence of this function leads to the phase diagram $T_{c}(p)$. In equilibrium, the interface free energy goes to zero as $T$ increases toward $T_{\text {ons }}$, and there is no surface tension in the disordered phase for $T>T_{\text {ons }}(28)$. Therefore, one may identify $T_{c}(p)$ as the (finite) temperature for which $\sigma_{0}(T, p)=0$. We thus obtain the result in Fig. 1. On the other hand, $\sigma_{0}$ is linear in $T$ as $T \rightarrow 0$ (see Fig. 4), namely $\sigma_{0}(T, p) \approx \alpha(p) T$, with (25)

$$
\alpha(p)=\ln \left(\frac{1-\sqrt{p}}{p+\sqrt{p}}\right) .
$$

The condition $\alpha\left(p_{c}\right)=0$ thus signals the onset of disorder at low temperature. This yields $p_{c}=(\sqrt{2}-1)^{2} \approx 0.1716$, in excellent agreement with previous MC simulations $(11 ;$ 19). 


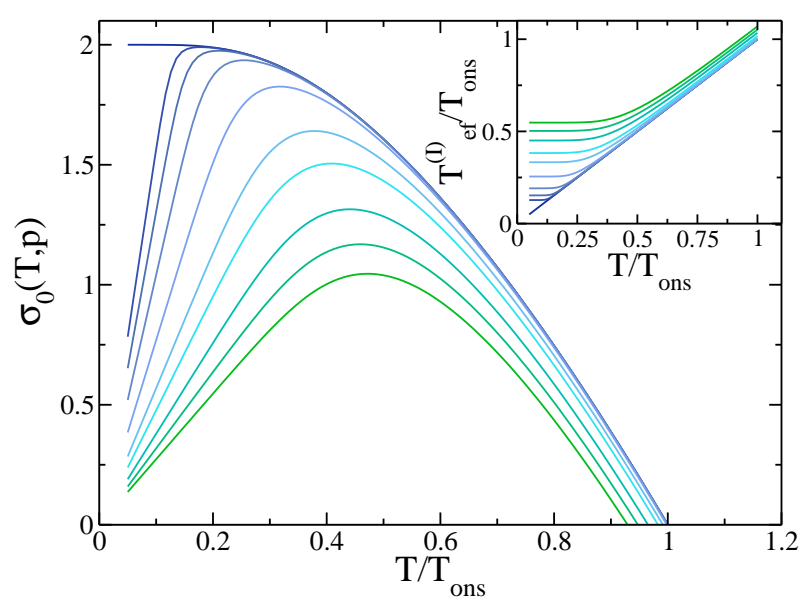

FIG. 4 (Color) The nonequilibrium surface tension as a function of $T$ for, from top to bottom, $p=0,10^{-6}, 10^{-5}, 10^{-4}, 10^{-3}, 5 \times$ $10^{-3}, 10^{-2}, 2 \times 10^{-2}, 3 \times 10^{-2}$, and $4 \times 10^{-2}$. For any $p>0$, the surface tension behaves non-monotonously, contrary to the equilibrium case. Inset: The effective interface temperature $T_{\mathrm{ef}}^{(\mathrm{I})}(T, p)$, as defined in the main text, versus $T$ for the same values of $\mathrm{p}$ than in the main graph. Notice that $T_{\mathrm{ef}}^{(\mathrm{I})}(T, p>0)$ strongly deviates from $T$ in the low-temperature regime.

\section{Effective Free-Energy and Nucleation}

The structural similarities between equilibrium and nonequilibrium excitations responsible for the metastable-stable transition allows one to write an effective free energy associated to a cluster of radius $R$. In formal analogy with the equilibrium case (므), we assume that

$$
\mathcal{F}(R)=\gamma\left[2 \Omega R \sigma_{0}-\Omega R^{2} 2 m_{0}|h|\right] .
$$

Here, $\sigma_{0}$ is the nonequilibrium surface tension in the previous subsection, $m_{0}(T, p)$ is the zero-field spontaneous magnetization, which follows from the mean-field scheme in $\$ I I . A$ and $\Omega(T, p)$ is the cluster form factor, which relates the cluster linear length $R$ to its volume, $C=\Omega R^{2}$. This may be computed from $\sigma_{0}$ via the Wulff construction (25; 29). The multiplicative factor in eq. (11), $\gamma \simeq 1$, is a phenomenological parameter, which might have a very weak dependence on $T$ and $p$, intended to capture small corrections to classical nucleation theory due to correlations between different clusters, inhomogeneous magnetization profiles within a cluster, etc (7).

The first term in $\mathcal{F}(R)$ measures the cost of the cluster interface, while the second term stands for the bulk gain. In this way, small clusters - with a large surface/volume ratio - tend to shrink, while large clusters tend to grow. The critical cluster radius, $\mathcal{R}_{c}$, which corresponds to the maximum of $\mathcal{F}(R)$, separates these two tendencies. One has that

$$
\mathcal{R}_{c}=\frac{\sigma_{0}}{2 m_{0}|h|} .
$$

We measured this quantity in MC simulations. With this aim, one produces an initial state with a single square cluster of down spins and side $2 R$ (the stable phase) in a sea of up spins. This is highly unstable, so that any subcritical initial cluster, $R<\mathcal{R}_{c}(T, p)$, will very quickly shrink, while supercritical ones, $R>\mathcal{R}_{c}(T, p)$, will rapidly grow to cover the whole system. Since our dynamics is stochastic, we define the probability that a cluster of size $R$ is supercritical, $P_{\mathrm{spc}}(R)$. This is measured in practice by simply repeating many times the simulation and counting the number of times that the initial cluster grows to cover the system. $\mathcal{R}_{c}$ is then defined as the solution of $P_{\mathrm{spc}}\left(\mathcal{R}_{c}\right)=0.5$. As illustrated in the inset to Fig. 囵, $P_{\mathrm{spc}}(R)$ shows a sharp transition from 0 to 1 , which allows for a relatively accurate estimate of $\mathcal{R}_{c} .{ }^{2}$ The agreement shown in Fig. 5 between these results and our analytical predictions is excellent for temperatures well below $T_{c}(p)$. Interesting enough, $\mathcal{R}_{c}(T, p>0, h)$ exhibits non-monotonous $T$-dependence, as expected from the anomalous low- $T$ behavior of $\sigma_{0}$.

\footnotetext{
${ }^{2}$ Our numerical results on $\mathcal{R}_{c}$ depend in principle on the shape of the initial excitation, which we choose squared. However, we checked that using spherical initial excitations (good at high $T$ ) one obtains estimates for $\mathcal{R}_{c}$ which are very close to those obtained with squared excitations (expected at low $T$, see Ref. (25)).
} 


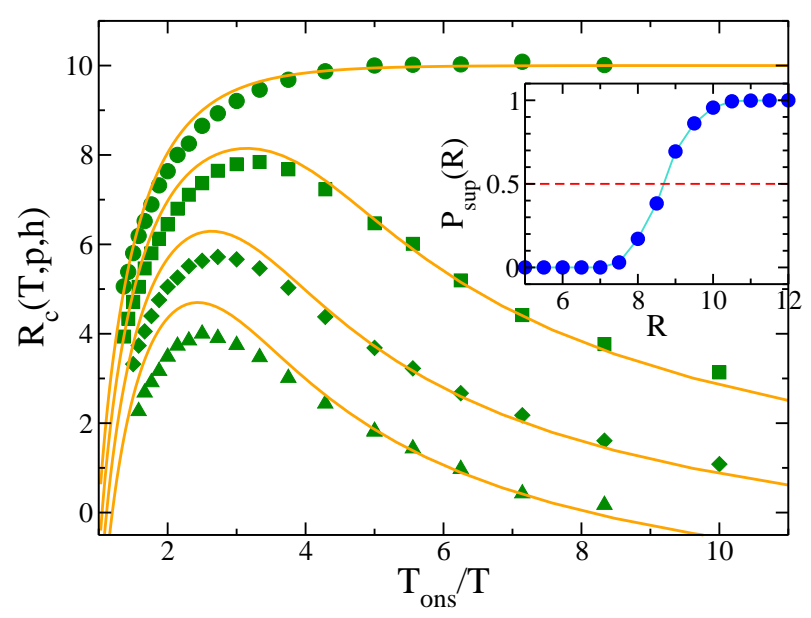

FIG. 5 (Color) $\mathcal{R}_{c}$ vs. $T^{-1}$ for $L=53, h=-0.1$ and, from top to bottom, $p=0,0.001,0.005$ and 0.01 . Symbols are MC results. Lines correspond to the theoretical prediction. The $n^{\text {th }}$ curve (from bottom to top) has been shifted (4- $n$ ) units in the $\hat{y}$-axis. Inset: $\mathrm{MC}$ results for $P_{\mathrm{spc}}(R)$ as a function of $R$ for $L=53$ at $T=0.4 T_{\mathrm{ons}}, p=0$, and $h=-0.1$. All the data in these graphs correspond to an average over 1000 independent experiments.

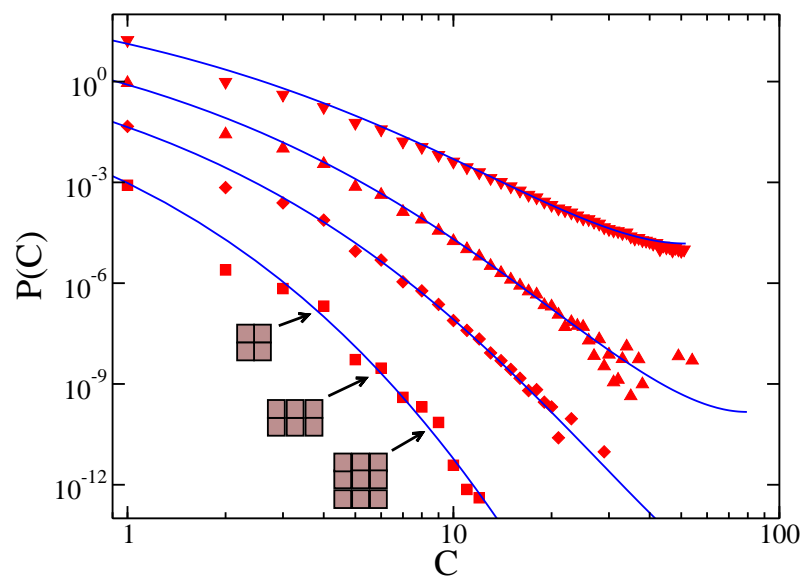

FIG. 6 (Color) Cluster distribution $P(C)$ for $T=0.2 T_{\text {ons }}, L=53, h=-0.1$ and, from bottom to top, $p=0.001,0.005,0.01$, 0.02 , with $\gamma=0.815,0.82,0.83,0.85$, respectively. Lines are theoretical predictions and points are MC results. The $n^{\text {th }}$ curve (from bottom to top) has been rescaled by a factor $10^{(n-1)}$ in the $\hat{y}$-axis.

Our ansatz in eq. (11) also implies that the fraction of stable-phase clusters of volume $C=\Omega R^{2}$ in the metastable phase follows a Boltzmann distribution

$$
P(C)=\mathcal{M}^{-1} \exp \left[-2 \beta \gamma\left(\sigma_{0} \sqrt{\Omega C}-m_{0}|h| C\right)\right]
$$

Here, the normalization $\mathcal{M}=2 \Theta /(1-m)$, where $\Theta=\sum_{C=1}^{C_{*}} C \exp [-\beta \mathcal{F}(C)]$ with $C_{*}=\Omega \mathcal{R}_{c}^{2}$, is defined so that the metastable state has magnetization $m(T, p, h)$ as derived in $₫$ III.A. The cluster distribution $P(C)$ which is obtained in $\mathrm{MC}$ simulations for times much shorter than the relaxation time is shown in Fig. 6. This compares very well with the analytical prediction, eq. (13). For $p=0.001$, we observe a non-trivial structure in $P(C)$ which is not captured by our continuous description. This is due to the underlying lattice anisotropy, which for low $-T$ and small $p$ gives rise to resonances in $P(C)$ for clusters with complete shells, i.e. $2 \times 2,3 \times 2,3 \times 3$; see Fig. 6, For larger $p$ and/or $T$, fluctuations smear out these resonances. Notice in Fig. [6 that the nonequilibrium perturbation $p$ enhances fluctuations and favors larger clusters. Similar good agreement for $P(C)$ is found in a wide range of values for $T, p$ and $h$. On the other hand, systematic corrections in $P(C)$ show up for microscopic clusters. These appear because our main ansatz, eq. (11), is based on mesoscopic considerations and therefore is only valid for large enough clusters. The observed deviations won't play however a relevant role in our discussion, as far as the critical cluster remains large enough, as it is the case here, see Fig. 5.

The assumption above on $\mathcal{F}(R)$ implies a force per unit area which controls the growth of supercritical clusters. It 


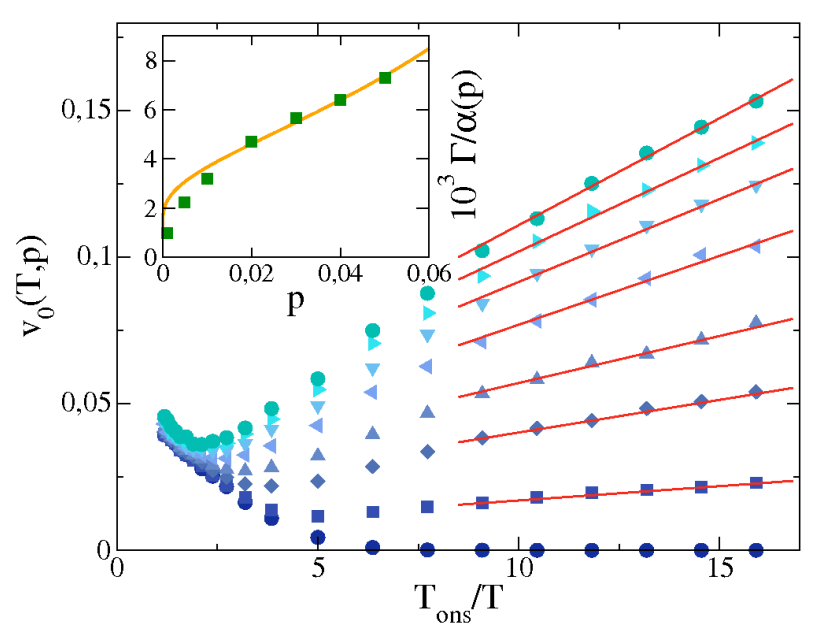

FIG. 7 (Color) Propagation velocity $v_{0}$ vs. $T^{-1}$ for $h=-0.1$ and $p=0,0.001,0.005,0.01,0.02,0.03,0.04,0.05$ (from bottom to top). Curves are linear fits to data. Inset: Slope of linear fits vs. $p$. The line is the theoretical prediction.

follows that the propagation velocity of a cluster in the large-size limit will obey the Allen-Cahn expression (4; ; 5 ):

$$
v_{0}=\frac{2 \Gamma^{\prime} m_{0}|h|}{\sigma_{0}}
$$

where $\Gamma^{\prime}$ is a non-universal constant. We measured $v_{0}$ in MC simulations of an infinitely-large cluster. This was implemented in practice by a flat interface separating the stable phase from the metastable one which constantly propagates. The results, which are shown in Fig. 7 agree with our predictions. We also observe that $v_{0}$ exhibits non-monotonous $T$-dependence, as expected given the behavior described above for $\sigma_{0}$. This implies, in particular, that, at low $T$, cooling the system favors the interface propagation or, in other words, that the interface dynamics becomes more sluggish as we raise the temperature in the low- $T$ regime.

As discussed before, $\sigma_{0}$ increases linearly with $T$ at low temperature, namely, $\sigma_{0} \approx \alpha(p) T$ as $T \rightarrow 0$ with $\alpha(p)$ given in eq. (10). Therefore we expect in this regime that $v_{0} \approx[\Gamma / \alpha(p)]\left(T_{\text {ons }} / T\right)$ with $\Gamma=2 \Gamma^{\prime}|h| T_{\text {ons }}^{-1}$ assuming that $m_{0}(T \rightarrow 0, p) \sim \mathcal{O}(1)$. This low $-T$ behavior, linear in $T^{-1}$, is also confirmed by simulations, see the inset to Fig. 7 , with $\Gamma \approx 0.0077$.

The nucleation rate $\mathcal{I}$, i.e., the probability that a critical cluster nucleates per unit time and per unit volume, may be written as

$$
\mathcal{I}=A|h|^{\delta} \exp \left[-\beta \mathcal{F}\left(\mathcal{R}_{c}\right)\right],
$$

where $A(p)$ is a non-universal amplitude and $\delta \approx 3$ for random updatings $(\underline{6})$. Two main relaxation patterns arise depending on the interplay between the relevant length scales in the problem. In fact, one needs to deal with the competition between the system size, $L$, and the mean cluster separation, $\mathcal{R}_{0}(T, p, h)$. The two other important length scales, namely the critical radius, $\mathcal{R}_{c}$, and the thermal correlation length in the metastable phase, $\xi_{\mathrm{ms}}$, do not compete with the former two given that they are much smaller in the most interesting regime, $\xi_{\mathrm{ms}} \ll \mathcal{R}_{c} \ll \mathcal{R}_{0}, L$. Now $\mathcal{R}_{0}$ can be calculated together with its associated time scale $t_{0}$ by requiring that $\mathcal{R}_{0}=v_{0} t_{0}$, which states that a supercritical cluster propagating with velocity $v_{0}$ will grow a distance $\mathcal{R}_{0}$ in a time $t_{0}$, and that $\mathcal{R}_{0}^{2} t_{0} \mathcal{I}=1$, which states that the probability of nucleating a volume $\mathcal{R}_{0}^{2}$ in a time $t_{0}$ is (by definition) one. Therefore, $\mathcal{R}_{0}=\left(v_{0} / \mathcal{I}\right)^{1 / 3}$. For $\mathcal{R}_{0} \gg L$, the random nucleation of a single critical cluster is the relevant excitation. In this regime, known as Single-Droplet (SD) region, the lifetime of the metastable state is $\tau_{\mathrm{SD}}=\left(L^{2} \mathcal{I}\right)^{-1}$. On the other hand, for $\mathcal{R}_{0} \ll L$, the metastable-stable transition proceeds via the nucleation of many critical clusters, a regime known as Multi-Droplet (MD) region, and $\tau_{\mathrm{MD}}=\left[\Omega v_{0}^{2} \mathcal{I} /(3 \ln 2)\right]^{-1 / 3}(\underline{6})$. Summing up,

$$
\tau(T, p, h) \propto \begin{cases}\left(L^{2} \mathcal{I}\right)^{-1} & \mathcal{R}_{0} \gg L(\mathrm{SD}), \\ \left(v_{0}^{2} \mathcal{I}\right)^{-1 / 3} & \mathcal{R}_{0} \ll L(\mathrm{MD}) .\end{cases}
$$

The crossover between these regimes, given by the condition $\mathcal{R}_{0}=L$, characterizes the dynamic spinodal point, namely,

$$
\left|h_{\mathrm{DSP}}\right|(T, p)=\frac{\Omega \gamma \sigma_{0}^{2}}{6 m_{0} T \ln L}
$$




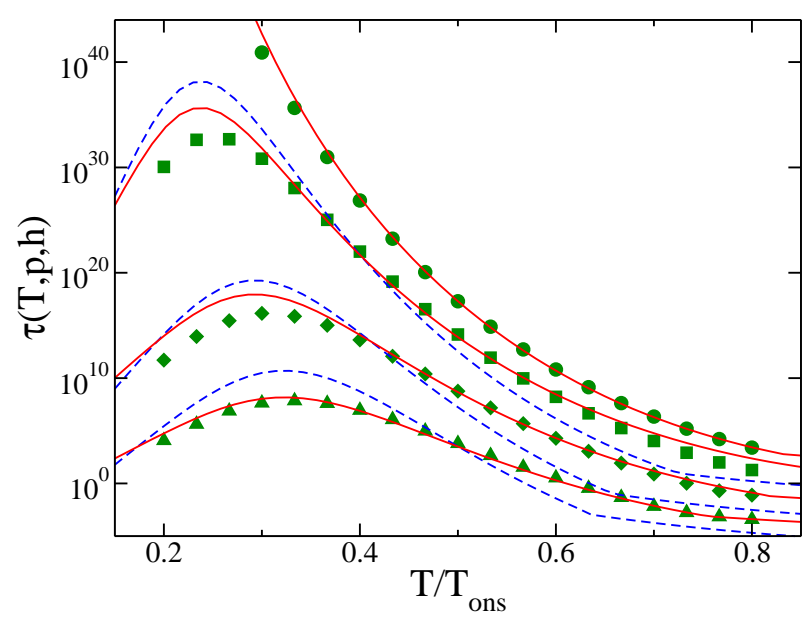

FIG. 8 (Color) Lifetime $\tau$ of the metastable state vs. $T$ for the same values of $p$ and conditions than in Fig. 5 . The $n^{\text {th }}$ curve (from top to bottom) is rescaled by a factor $10^{-2(n-1)}$. Solid lines are theoretical predictions for (from top to bottom) $\gamma=1,0.85,0.77,0.65$. Amplitudes $A(p)$ are in the range $\left[10^{-3}, 10^{-2}\right]$. Dashed lines are predictions for $\gamma=1$.

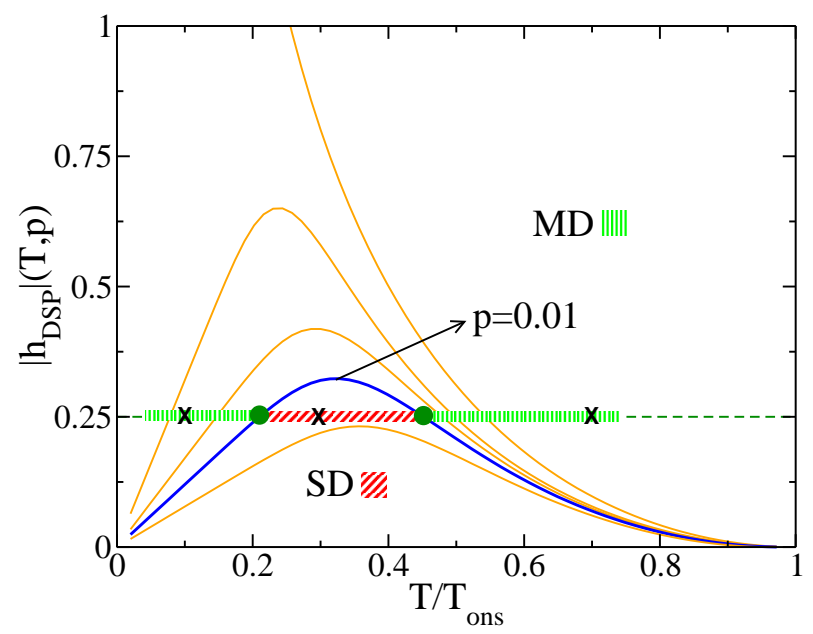

FIG. 9 (Color) The dynamic spinodal $\left|h_{\mathrm{DSP}}\right|$, as defined in eq. (17), versus $T$ for $p=0,0.001,0.005,0.01$, and 0.02 from top to bottom, respectively. We explicitly indicate the $T$ ranges corresponding to the SD and MD regimes in the case of the example in Fig. 3. namely, for $|h|=0.25$ and $p=0.01$. The symbols $\times$ mark the temperatures $T / T_{\text {ons }}=0.1,0.3$ and 0.7 for $|h|=0.25$. Notice that, unlike at equilibrium, there is here a low- $T$ regime where the MD scenario holds. This is confirmed in Fig. 3

That is, one should observe the SD regime for $|h|<\left|h_{\mathrm{DSP}}\right|$, while the MD regime is expected to dominate otherwise.

We estimated $\tau$ from MC simulations by defining it as an average of the first-passage time to zero magnetization. Due to the strong local stability that characterizes the metastable state at low temperature, $\tau$ may be extremely large in practice. (For example, this goes up to $10^{40} \mathrm{MC}$ step per spin in Fig. 8 . Assuming the MC time unit corresponds to the inverse of the typical phonon frequency ${ }^{3}$, which is of order of $10^{-12} \mathrm{~s}$, this corresponds to $\tau \sim 10^{28} \mathrm{~s}$ !). Therefore we needed to use an efficient rejection-free algorithm. Our choice was the $s-1$ variant of the MC algorithm with absorbing Markov chains, together with the slow-forcing approximation (31). Fig. 8 shows our results for $\tau$ vs. $T$ and different values of $p$, together with theoretical predictions. Interesting enough, we observe that $\tau$ increases with $T$ at low- $T$ for fixed $p>0$. That is, the local stability of nonequilibrium metastable states is enhanced at low- $T$ as the strength of the thermal noise $(T)$ increases. This, which is in contrast with the Arrhenius curve observed in equilibrium, closely resembles the noise-enhanced stability (NES) phenomenon reported in experiments on unstable

\footnotetext{
${ }^{3}$ Certain rare-earth magnetic materials can be modeled to a good approximation by the Ising Hamiltonian (30). When we talk here about the phonon frequency we refer to the phonon frequency in one of these real magnetic system with Ising-like properties.
} 
systems (32). On the other hand, increasing $p$ for fixed $T$ always results in shorter $\tau$. This complex phenomenology is captured by our simple ansatz eq. (11), which traces back the NES phenomenon to the low $-T$ anomaly in $\sigma_{0}$, which in turn is a nonlinear cooperative effect due to the interplay between thermal and nonequilibrium fluctuations. That is, though any of these two noise sources ( $T$ and $p$ ) will separately induce disorder, their combined effect results in (resonant) stabilization of the nonequilibrium metastable state.

This interesting effect is also evident in the morphology of the metastable-stable transition. In particular, $\left|h_{\mathrm{DSP}}\right|$ inherits the non-monotonous $T$-dependence of $\sigma_{0}$, see Fig. 9, resulting in a novel MD regime at low- $T$ not observed in equilibrium. For instance, for $h=-0.25$ and $p=0.01$, we expect from Fig. 9 MD morphology for, e.g., $T=0.1 T_{\text {ons }}$ and $T=0.7 T_{\text {ons }}$ but SD behavior for $T=0.3 T_{\text {ons }}$, which is confirmed by direct inspection of escape configurations such as the ones in Fig. 3. Notice that the SD decay mechanism is a finite-size effect, and that MD will be the only relevant mechanism for large enough $L$. However, the relevance of the SD regime vanishes logarithmically with $L$ - see eq. (17) - so that it could be observable in mesoscopic samples, at least (6)).

\section{Langevin Type Description}

We may get further insight into the above stochastic resonance phenomena and the peculiar low- $T$ behavior by rewriting the rate (2) as

$$
p+(1-p) \Psi\left(\beta \Delta \mathcal{H}_{i}\right) \equiv \Psi\left(\beta_{\mathrm{ef}} \Delta \mathcal{H}_{i}\right)
$$

The resulting effective temperature $T_{\text {ef }} \equiv \beta_{\text {ef }}^{-1}$ then follows as

$$
T_{\mathrm{ef}}\left(T, p ; \Delta \mathcal{H}_{i}\right)=\frac{T \Delta \mathcal{H}_{i}}{\Delta \mathcal{H}_{i}+T \ln \left(\frac{1-p}{1+p \mathrm{e}^{\beta \Delta \mathcal{H}_{i}}}\right)}
$$

For any $p>0, T_{\text {ef }}$ changes from spin to spin and depends on the local order as characterized, for instance, by the number $\eta_{i}$ of neighbors that are in the same state than the central spin (in fact, one can write $\Delta \mathcal{H}_{i}=4\left(\eta_{i}-2\right)+2 s_{i} h$, see Table I): the larger $\eta_{i}$, the higher the amount of local order, and the larger the local effective temperature. This observation is not restricted to the Glauber function we use for $\Psi$ in this paper, see $\S \mathrm{II}$ and eq. (2), but holds for many other known forms for $\Psi$. For instance, for the Metropolis case, which is analytically simpler for the purpose, one obtains for $h \rightarrow 0$ that

$$
T_{\mathrm{ef}}^{\left(\eta_{i}\right)}(T, p)= \begin{cases}-8\left(\ln \left[p+(1-p) \mathrm{e}^{-8 \beta}\right]\right)^{-1} & \eta_{i}=4, \\ -4\left(\ln \left[p+(1-p) \mathrm{e}^{-4 \beta}\right]\right)^{-1} & \eta_{i}=3, \\ T & \eta_{i}=2,1,0\end{cases}
$$

such that $T_{\mathrm{ef}}^{(4)} \geq T_{\mathrm{ef}}^{(3)} \geq T_{\mathrm{ef}}^{(2,1,0)}>0$, the equality holding only in the equilibrium limit $p=0$. Therefore, in all cases, the strength of fluctuations that affect a spin increases with the local order parameter. This is the fingerprint of multiplicative noise, which suggest us to write a Langevin equation with the hope it will capture the physics of the problem. Defining the coarse-grained order parameter as the local magnetization field $\psi(\mathbf{r}, t)$, we propose the following Langevin equation

$$
\frac{\partial \psi(\mathbf{r}, t)}{\partial t}=D \nabla^{2} \psi(\mathbf{r}, t)+\psi(\mathbf{r}, t)-\psi(\mathbf{r}, t)^{3}+h+\sqrt{D+\mu \psi(\mathbf{r}, t)^{2}} \xi(\mathbf{r}, t)
$$

where $\xi(\mathbf{r}, t)$ is a Gaussian white noise with $\langle\xi(\mathbf{r}, t)\rangle=0$ and $\left\langle\xi(\mathbf{r}, t) \xi\left(\mathbf{r}^{\prime}, t^{\prime}\right)\right\rangle=2 \delta\left(t-t^{\prime}\right) \delta\left(\mathbf{r}-\mathbf{r}^{\prime}\right), D$ is the strength of the thermal (additive) noise, $h$ is an external field parameter, and $\mu$ is the renormalized version of the nonequilibrium parameter $p$. This equation describes the coarse-grained dynamics of a Ising-like system in the broken-symmetry phase under an external magnetic field, with a noise term whose amplitude increases as the local order parameter $\psi(\mathbf{r}, t)$ increases, in close analogy with our observations above. Solving the full spatially-dependent problem, eq. (21), is a complex endeavor which we will not undertake here. However, as a proof of concept, one can analytically solve a simplified model corresponding to the mean-field, 0-dimensional form of the above Langevin equation:

$$
\frac{\partial \psi}{\partial t}=\psi-\psi^{3}+h+\sqrt{D+\mu \psi^{2}} \xi(t),
$$

where now $\psi(t)$ has no spatial dependence. Despite the obvious simplifications, this equation still contains the essential competition between thermal $(D)$ and nonequilibrium $(\mu)$ fluctuations in a metastable potential that characterizes 


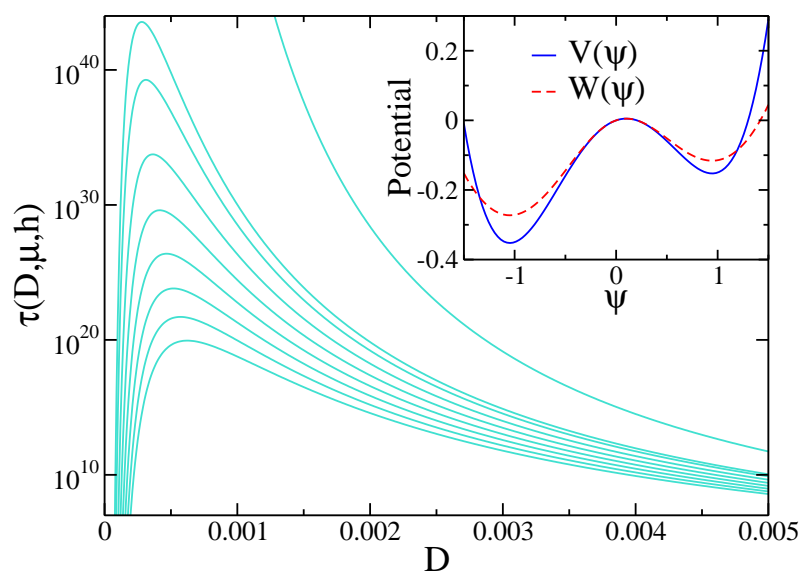

FIG. 10 (Color) Lifetime $\tau$, as derived from the Langevin equation (22), versus $D$ for $h=-0.1$ and for $\mu \in\left[0,6 \times 10^{-3}\right]$ increasing from top to bottom. The inset illustrates the potential $V$ and the effective potential $W$ as defined in the main text.

our system ${ }^{4}$. In particular, eq. (22) describes the motion of a Brownian particle in an asymmetric bimodal potential, namely, $V(\psi)=-\frac{1}{2} \psi^{2}+\frac{1}{4} \psi^{4}-h \psi-$ which is depicted in the inset to Fig. 10. The particle is subject to fluctuations which increase with $\psi^{2}$ and whose amplitude remains non-zero as $D \rightarrow 0$ for any $\mu>0$. Notice that the noise strength $\sqrt{D+\mu \psi^{2}}$ increases with the order parameter $\left(\sim \psi^{2}\right)$, in accordance with our observation above concerning eqs. (19)-(20). For $\mu=0$, this reduces to the additive theory which describes simple symmetry-breaking systems (33) while, for $\mu>0$, additive and multiplicative fluctuations compete in eq. (22).

The Stratonovich stochastically-equivalent Fokker-Planck equation of eq. (22) is $\partial_{t} P(\psi, t)=-\partial_{\psi}[A(\psi) P(\psi, t)]+$ $\frac{1}{2} \partial_{\psi}^{2}[B(\psi) P(\psi, t)]$ with $A(\psi)=(1+\mu) \psi-\psi^{3}+h$ and $B(\psi)=2\left(D+\mu \psi^{2}\right)$. This yields the steady distribution

$$
P_{\mathrm{st}}(\psi)=\frac{\Lambda}{2 \sqrt{\mu\left(D+\mu \psi^{2}\right)}} \exp \left[-\frac{W(\psi)}{D}\right],
$$

where $\Lambda$ is a normalization constant, and

$$
W(\psi)=\frac{D}{\mu}\left[\frac{1}{2} \psi^{2}-\frac{1}{2}\left(1+\frac{D}{\mu}\right) \ln \left(\frac{D}{\mu}+\psi^{2}\right)-h \sqrt{\frac{\mu}{D}} \tan ^{-1}\left(\sqrt{\frac{\mu}{D}} \psi\right)\right] .
$$

The extrema of the effective potential $W(\psi)$ are the same than those of $V(\psi)$, namely $\psi_{k}=2 \cos \left(\theta_{k}\right) / \sqrt{3}$, with $\theta_{k}=\frac{1}{3}\left[\cos ^{-1}\left(-\frac{1}{2} \sqrt{27} h\right)+2 k \pi\right]$, and $k=0,1,2$, see inset to Fig. [10 and Fig. 11]. For $h<0, \psi_{0}, \psi_{1}$ and $\psi_{2}$ correspond to the metastable, stable and unstable extrema, respectively, and the escape time from the metastable minimum is $(\underline{33})$

$$
\tau(D, \mu, h) \approx \frac{2 \pi}{\sqrt{\left|V^{\prime \prime}\left(\psi_{0}\right) V^{\prime \prime}\left(\psi_{2}\right)\right|}} \exp \left[\frac{1}{D}\left(W\left(\psi_{2}\right)-W\left(\psi_{0}\right)\right)\right]
$$

where $V^{\prime \prime}=\partial_{x}^{2} V(x)$. Identifying $D$ with the temperature parameter in our microscopic model, this precisely reproduces the thermal NES phenomenon for $\tau$ as described above; compare Figs. 10 and 8 . Therefore, the resonant, temperature-enhanced stabilization of nonequilibrium metastable states may be regarded as a consequence of competition between additive and multiplicative noise.

\section{E. The Limit of Metastability}

When the magnetic field $|h|$ is increased, the strength of the metastable state decreases. That is, the local minimum in the free-energy functional associated to the metastable phase becomes less pronounced. Upon further increasing $|h|$,

\footnotetext{
${ }^{4}$ As a result of the homogeneity hypothesis associated to the mean-field approximation, eq. (22) has no spatial structure and therefore is unable to describe the compact excitations responsible of the metastable state decay in the microscopic model. Despite this obvious difficulty, eq. (22) still contains the relevant competition between additive and multiplicative fluctuations which gives rise to resonant behavior in this model.
} 


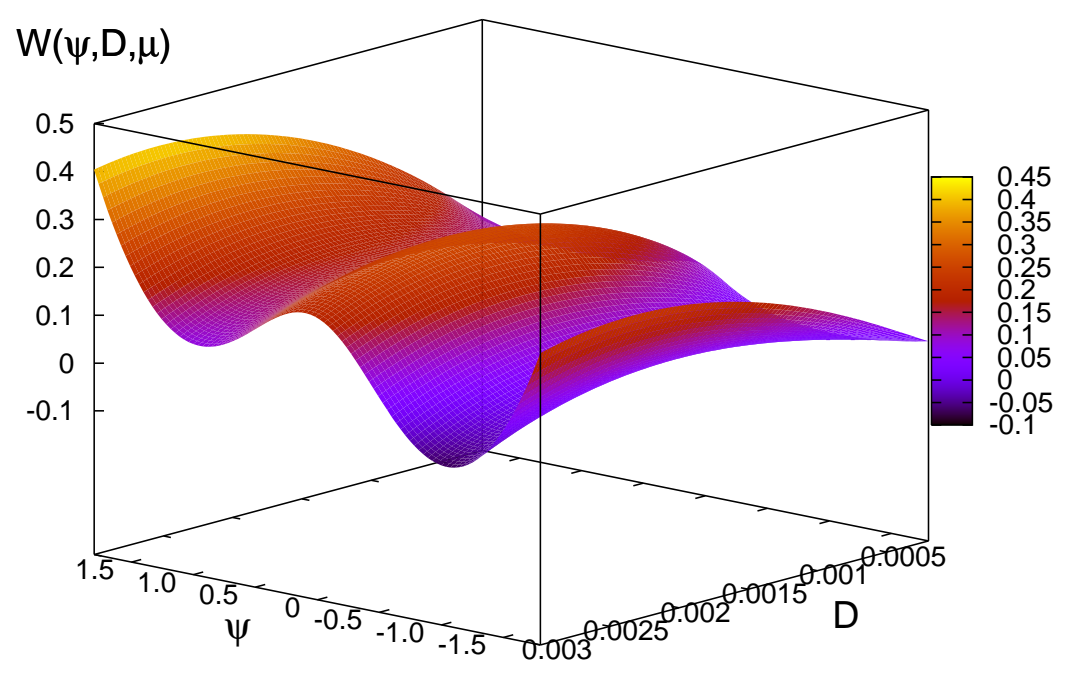

FIG. 11 (Color) Effective potential $W(\psi, D, \mu)$ for $\mu=4 \times 10^{-3}$ and $h=-0.1$. Note how the energy barrier between the two local minima changes as a function of $D$ for this $\mu>0$.

such local minimum eventually disappears. At this point, the metastable state becomes unstable, which means that its relaxation toward the final stable configuration is no longer hampered by any free-energy barrier. The magnetic field strength $h^{*}(T, p)$ at which this metastable-unstable transition occurs is known as the spinodal (34) or, sometimes (see Appendix A) pseudospinodal or intrinsic-coercive field.

As $|h|$ is increased, the metastable phase remains homogeneous on a coarse-grained length scale, for times shorter than $\tau$, so that the mean-field approach in section III.A is suitable within this context. The locally-stable steady magnetization, as computed from the stationary solution of eqs. (4) and (5), exhibits two branches as a function of $h$, see the inset in the left graph of Fig. 12. This hysteresis loop reveals that, at the mean-field level, metastability disappears abruptly for any $|h|>h^{*}$. In order to evaluate $h^{*}(T, p)$, one may study how the metastable state responds to small perturbations of the applied field. If $\left(x_{\mathrm{st}}^{\mathrm{h}_{0}}, z_{\mathrm{st}}^{\mathrm{h}_{0}}\right)$ is a locally-stable stationary state for $T, p$ and $h_{0}$, with magnetization opposed to $h_{0}$, and we perturb $h=h_{0}+\delta h$, the new locally-stable stationary solution is modified according to $x_{\mathrm{st}}^{\mathrm{h}}=x_{\mathrm{st}}^{\mathrm{h}_{0}}+\epsilon_{\mathrm{x}}$ and $z_{\mathrm{st}}^{\mathrm{h}}=z_{\mathrm{st}}^{\mathrm{h}_{0}}+\epsilon_{\mathrm{z}}$. One obtains to first order that

$$
\epsilon_{\mathrm{x}}=\left[\frac{\partial_{h} G_{2} \partial_{z} G_{1}-\partial_{h} G_{1} \partial_{z} G_{2}}{\partial_{x} G_{1} \partial_{z} G_{2}-\partial_{x} G_{2} \partial_{z} G_{1}}\right]_{0} \times \delta h
$$

and a similar expression for $\epsilon_{\mathrm{z}}$, where the quantity in brackets is evaluated at $\left(x_{\mathrm{st}}^{\mathrm{h}_{0}}, z_{\mathrm{st}}^{\mathrm{h}_{0}}\right)$ for given values of the parameters $T, p$, and $h_{0}$, and the functions $G_{1,2}(x, z)$ are defined in eqs. (4) and (5). This (linear) response diverges for

$$
\left[\partial_{x} G_{1} \partial_{z} G_{2}-\partial_{x} G_{2} \partial_{z} G_{1}\right]_{0}=0
$$

which corresponds to a discontinuity in the metastable magnetization as a function of $h$. For fixed $T$ and $p$, the field for which eq. (27) holds is $h^{*}$. The left graph of Fig. 12 shows the mean-field result for $h^{*}(T, p)$ as obtained numerically from eq. (27). In particular, for $p=0$, we recover the standard equilibrium mean-field spinodal curve: Converging to 2 as $T \rightarrow 0$, linearly decreasing with temperature for small $T$, and vanishing as $\left(T_{\text {bethe }}-T\right)^{3 / 2}$ at the mean-field equilibrium critical point. For $p>0$, an instability separates two different low- $T$ behaviors of $h^{*}(T, p)$ depending on the amplitude of the nonequilibrium fluctuations. For small enough values of $p$, namely, $p \in[0,0.031]$, which includes the equilibrium case, the field $h^{*}(T, p)$ monotonously grows and extrapolates to 2 as $T \rightarrow 0$. For larger $p$, up to $p=5 / 32$, however, $h^{*}(T, p) \rightarrow 0$ as $T \rightarrow 0$, with a maximum at some intermediate value of $T$.

The value $p=\pi_{c} \approx 0.0315$ separates these two types of asymptotic behavior. The $p<\pi_{c}$ regime can be understood on simple grounds. In this case, $h^{*}(T, p)$ increases as $T$ drops, meaning that a stronger field is needed to destroy metastability. In a metastable state, the tendency to maintain spin order prevails over the tendency of the individual spins to follow the external field. Since both $T$ and $p$ induce disorder, one should expect that decreasing $T$ and/or $p$ a stronger field will be needed to destroy the metastable state, as confirmed in Fig. 12 for $p<\pi_{c}$. 

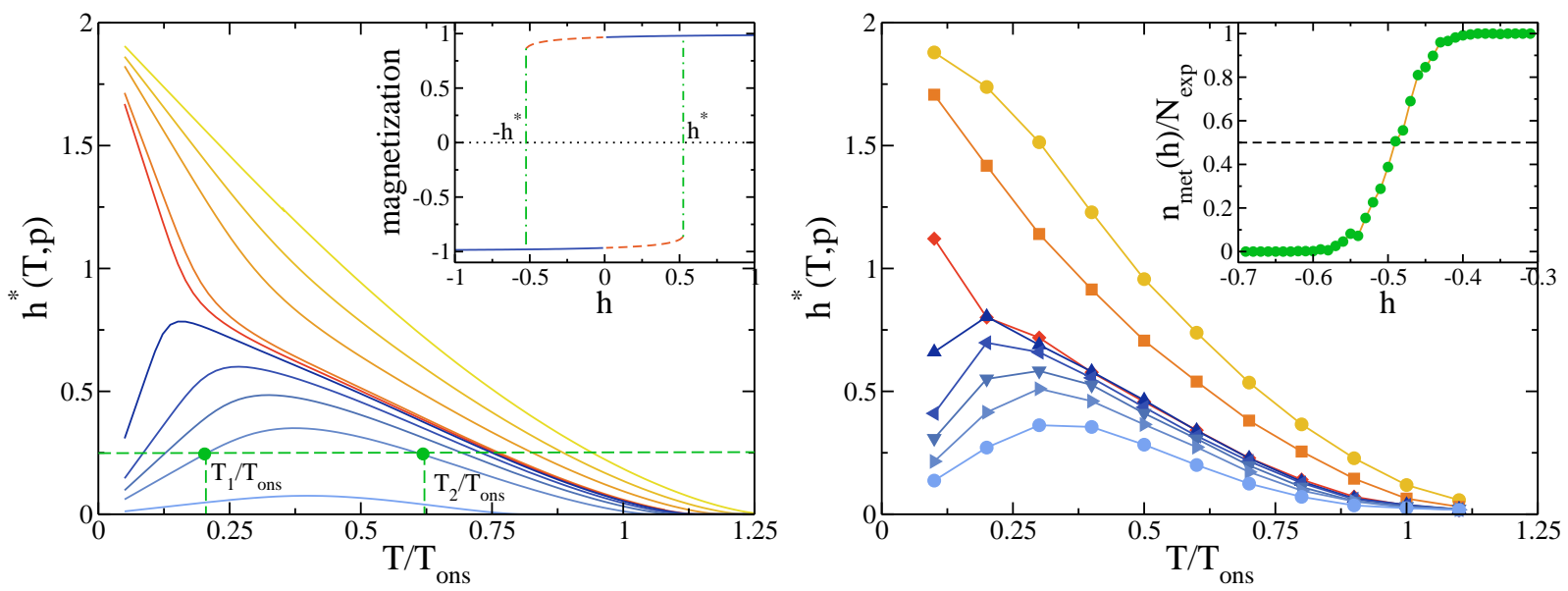

FIG. 12 (Color) Left panel: Mean-field result for $h^{*}(T, p)$ as a function of $T$ for, from top to bottom, $p=0,0.01,0.02$, $0.03,0.031,0.032,0.035,0.04,0.05$ and 0.1 . The qualitative change of behavior in the low temperature region occurs for $p \in(0.031,0.032)$. We also indicate for $|h|=0.25$ the temperatures $T_{1}<T_{2}$ which, as discussed in the main text, comprise the metastable region for $p=0.05$. The inset shows the two locally-stable steady magnetization branches as a function of $h$ for $T=0.7 T_{c}(0)$ and $p=0.005$. The solid (dashed) line represents stable (metastable) states. The dot-dashed line signals the discontinuous transition, at $h=h^{*}(T, p)$, where metastable states disappear. Right panel: Monte Carlo results for $h^{*}(T, p)$ as a function of $T$ for $L=53$ and, from top to bottom, $p=0,0.01,0.03,0.0305,0.0320,0.0350,0.04$ and 0.05 . The change of asymptotic behavior in the low- $T$ limit happens for $p \in(0.03,0.0305)$. The inset shows the probability of occurrence of metastability, as defined in the main text, as a function of $h<0$ for $L=53, T=0.7 T_{\text {ons }}$ and $p=0$. Data here correspond to an average over 500 independent demagnetization runs for each value of $h$.

The $p>\pi_{c}$ regime is more intriguing. Consider, for instance, $p=0.05>\pi_{c}$ and $|h|=0.25$. According to the left graph in Fig. 12, we can define two temperatures such that metastability only occurs for $T \in\left[T_{1}, T_{2}\right]$. The fact that $h^{*}$ goes to zero as $T \rightarrow 0$ in this regime means that, at low temperature, the nonequilibrium fluctuations are strong enough to destroy on their own the metastable phase. Based on the above argument, one would expect that increasing $T$ should avoid metastability. However, there is metastability for intermediate temperatures, $T \in\left[T_{1}, T_{2}\right]$. This reentrant behavior of the spinodal field suggests once more a resonance between thermal and nonequilibrium fluctuations: despite the intrinsic disordering effect of both noise sources, $T$ and $p$, they cooperate to produce metastability, for which local order prevails. We measured $h^{*}$ in MC simulations, confirming this; see the right graph in Fig. 12 and appendix A. In particular, we find the instability for $p=\pi_{c} \approx 0.03025$ in simulations, which is very close to the mean-field result.

There is an important difference between the two resonant phenomena above, i.e., the noise-enhanced stabilization of nonequilibrium metastable states and the reentrant behavior of the spinodal field. That is, while the former occurs for any $p>0$, the latter only occurs for $p>\pi_{c}$. In order to understand this fact, we studied the limit of metastability within the cluster nucleation scheme of section III.C In this case, $h^{*}(T, p)$ may be estimated from the condition that the critical cluster contains one single spin, $2 \mathcal{R}_{c}\left(T, p, h^{*}\right)=1$. This is equivalent to require that any microscopic fluctuation can trigger the transition toward the stable state, which signals the onset of instability. The result, namely, $h^{*}(T, p)=\sigma_{0} / m_{0}$ according to eq. (12), must be taken with some care, since it assumes that the surface tension $\sigma_{0}$, a macroscopic concept, is relevant for microscopic clusters. In fact, this corresponds to a mean-field spinodal $(\underline{6})$, which exhibits reentrant behavior for any $p>0$. Notice that the nonequilibrium perturbation in our model is stochastic in origin, producing random spin flips with probability $p$. For a macroscopically large interface the probability that these stochastic perturbations do not affect its structure and dynamics is negligible, so the nonequilibrium perturbations are always present, inducing the observed low- $T$ anomaly in $\sigma_{0}$, see $₫$ II.B. On the other hand, microscopic clusters (as those involved in the computation of $h^{*}$ ) have a non-negligible probability of not being affected by the stochastic perturbation $p$ during their nucleation and evolution for small enough $p$. In this way, these small clusters unaffected by $p$ would behave effectively as equilibrium clusters. This would renormalize the (microscopic) critical cluster size to a new, larger effective value, which thus would modify our estimation of the spinodal field $h^{*}$. Hence one expects $h^{*}$ to behave as in equilibrium for small enough values of $p$, as is in fact observed, while for large values of $p$ (say $p>\pi_{c}$ ) even small clusters are affected by the nonequilibrium perturbation, involving reentrant behavior for $h^{*}$. The challenge remains to put this qualitative argument, which seems plausible, into more quantitative grounds. 

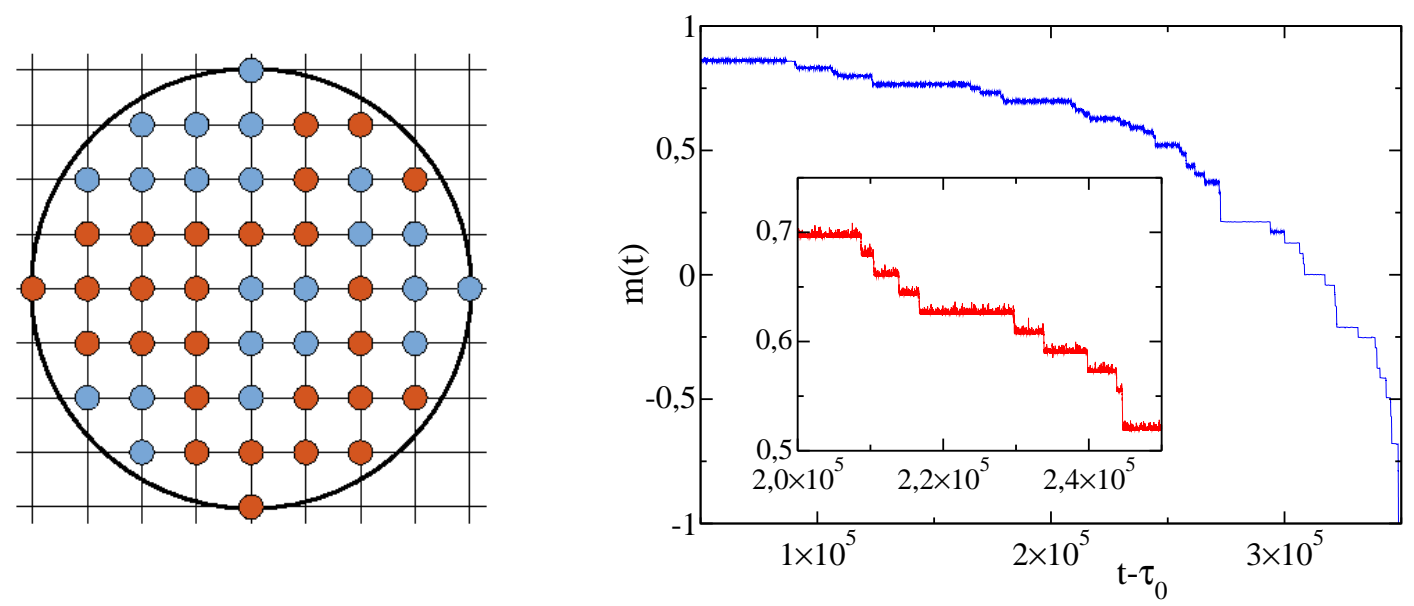

FIG. 13 (Color) Left: Schematic representation of the model particle with open circular boundary conditions. Spins at the border do not have nearest neighbor spins outside the circle. Right: Typical evolution in which the magnetization is observed to decay by jumps to the final stable state. This is for a single particle of radius $r=30\left(\sim 10^{3}\right.$ spins $)$ at low temperature, and for small values of $h$ and $p$ (see the main text). The time axis shows $t-\tau_{0}$ in MCSS (Monte Carlo steps per site) with $\tau_{0}=10^{30} \mathrm{MCSS}$; this is of order of the duration of the initial metastable state. The inset shows a significant detail of the relaxation.

\section{SMALL PARTICLES}

The above concerns bulk metastability, i.e., a property of macroscopic systems or models in the thermodynamic limit. However, the case of finite systems, in which surface effects are relevant, is of great interest. This occurs for magnetic particles in many applications, e.g., magnetic recording media where one often needs to deal with particle sizes ranging from mesoscopic to atomic levels, namely, clusters of $10^{4}$ to $10^{2}$ spins. We therefore also studied our model with open circular boundary conditions. The system is now defined on a square lattice, where we inscribe a circle of radius $r$, and assume that the bonds to any spin outside the circle are broken; see the left graph in Fig. 13.

The effects of free borders on the metastable-stable transition have already been studied in equilibrium systems (15; 17; 18). In this case, the system evolves to the stable state through the heterogeneous nucleation of one or several critical clusters, which always develop at the border, where it is energetically favorable. Apart from this, the properties of the metastable-stable transition do not change qualitatively as compared to the case of periodic boundaries. In our nonequilibrium system we also observe this kind of heterogeneous nucleation. However, in closer inspection, one notices that the randomness of the decay process is importantly enhanced, which results in some unexpected phenomenon.

The main observation is illustrated in the right graph of Fig. 13. That is, when observed on the appropriate time scale, namely, after each MC step (per spin), the time relaxation of the magnetization, $m(t)$, from the metastable state occurs by strictly monotonic changes. There is thus a sequence of well-defined abrupt jumps of $m(t)$, which we will name avalanches in the following, that resembles familiar relaxation processes in many complex systems in nature. We checked that this is a general feature of the model relaxation at low $T$. However, a too rapid relaxation and/or domains too diluted and interfaces too diffuse will tend to obscure the observation. Consequently, it turned out preferable to deal with small values of temperature, in order to have compact and well-defined clusters, and with values of $h$ that do not excessively accelerate the evolution. In addition, the parameter $p$ can take a wide range of values, provided that its effects are comparable to those of temperature. A perfect compromise is $T=0.11 T_{\text {ons }}$, $p=10^{-6}$, and $h=-0.1$, and most data below concern these parameter values.

\section{A. Avalanche Statistics}

To be precise, consider an avalanche that begins at time $t_{a}$, when the system magnetization is $m\left(t_{a}\right)$, and finishes at $t_{b}$. We define the avalanche duration as $\Delta_{t} \equiv\left|t_{a}-t_{b}\right|$ and its size as $\Delta_{m} \equiv\left|m\left(t_{a}\right)-m\left(t_{b}\right)\right|$, and we are interested on the associated respective distributions, $P\left(\Delta_{t}\right)$ and $P\left(\Delta_{m}\right)$. It turns out to be most important for the reported result to remove from the data some trivial extrinsic noise (35), namely, small thermal events of typical size (see appendix 

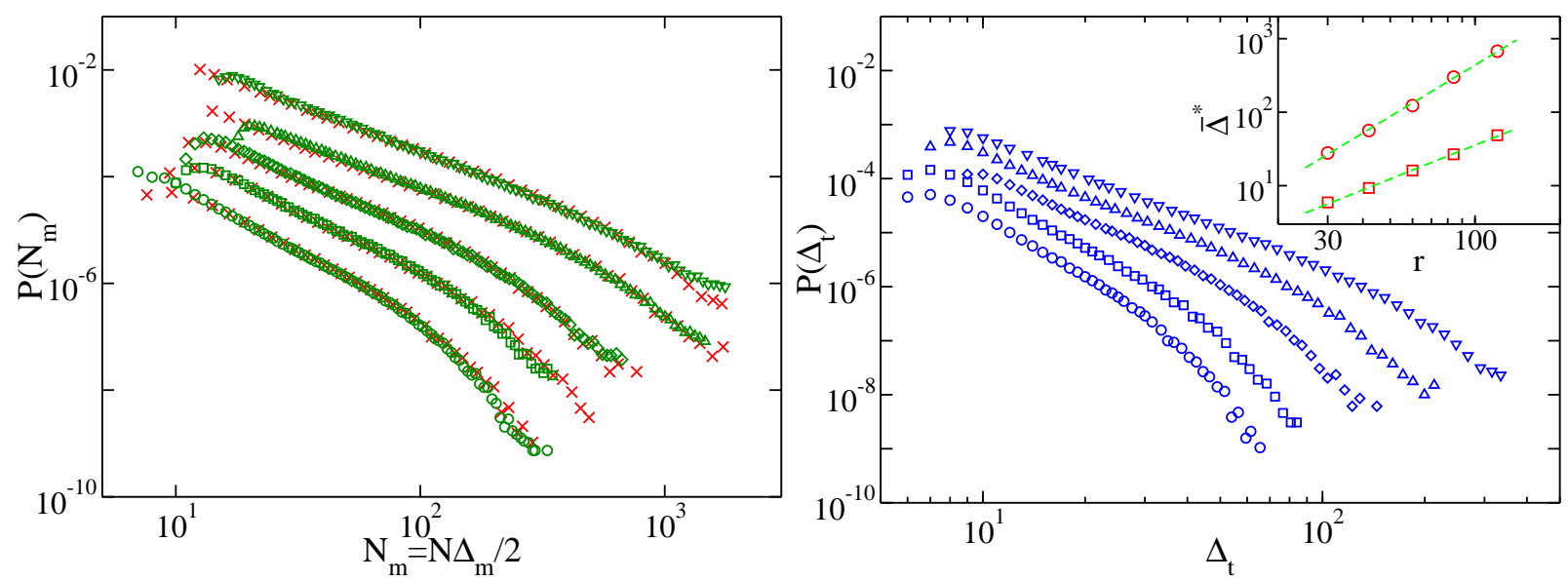

FIG. 14 (Color) Left: Log-log plot of the size distribution $P\left(\Delta_{m}\right)$ of avalanches (i.e., the data after subtracting "small events" as defined in the main text) for ensembles of independent model particles of radius (from bottom to top) $r=30,42,60,84$ and 120 , respectively. This corresponds to the $\mathrm{O}$ symbols (green). Plots of the corresponding duration distribution $P\left(\Delta_{t}\right)$ vs. $c \Delta_{t}^{\gamma}$ for each $r$ are also shown $(\times$, red) superimposed, with $c \approx 0.5$ and $\gamma \approx 1.52$ (see the main text). For visual convenience, the curves are shifted vertically by $4^{n}$ with $n=0$ to 4 from bottom to top. Right: Log-log plot of the duration distribution $P\left(\Delta_{t}\right)$ for the same ensembles. For visual convenience, these curves are shifted vertically by $2^{n}$ with $n=0$ to 4 from bottom to top. The inset shows a $\log -\log$ plot of the size (top) and duration (bottom) cutoffs $\bar{\Delta}^{*}$ vs. $r$. Lines are power-law fits. Running averages have been performed in all cases for clarity purposes.

B)

$$
\bar{\Delta}=\left\{\ln \frac{(1+p)\left[1+\mathrm{e}^{2 \beta|\mathrm{h}|}\right]}{p+\mathrm{e}^{2 \beta|\mathrm{h}|}}\right\}^{-1} .
$$

These events correspond to the short-length fluctuations that are evident by direct inspection in the inset of the right graph in figure 13. These also correspond to avalanches that originate at flat interfaces which, at low enough $T$, have a significant probability to form, as discussed below.

The distribution $P\left(\Delta_{m}\right)$ that results after deducting these small events is illustrated in the left graph of Fig. 14. This is well described by

$$
P\left(\Delta_{m}\right) \sim \Delta_{m}^{-\tau(r)},
$$

with a size-dependent exponent given by

$$
\tau(r)=\tau_{\infty}+a_{1} r^{-2},
$$

where $\tau_{\infty}=1.71(4)$. The left graph in Fig. 14 depicts the corresponding duration distributions. This follows

$$
P\left(\Delta_{t}\right) \sim \Delta_{t}^{-\alpha(r)},
$$

with

$$
\alpha(r)=\alpha_{\infty}+a_{2} r^{-2},
$$

where $\alpha_{\infty}=2.25(3)$. In both cases, size and duration, the apparent power law ends with an exponential tail, i.e.,

$$
P(\Delta) \sim \exp \left(-\Delta / \bar{\Delta}^{*}\right) .
$$

The cutoffs that we observe follow $\bar{\Delta}^{*} \sim r^{\beta}$ with $\beta_{m} \sim 2.32$ (6) and $\beta_{t} \sim 1.53$ (3), respectively (see the inset in the left graph of Fig. 14).

In practice, we observed this behavior for several combinations of values of the parameters $T, p$ and $h$. The requirement for an easy observation is that, as indicated above, the configurations need to be sufficiently compact and that none of the underlying processes should predominate so that it obscures the other. That is, the above behavior 

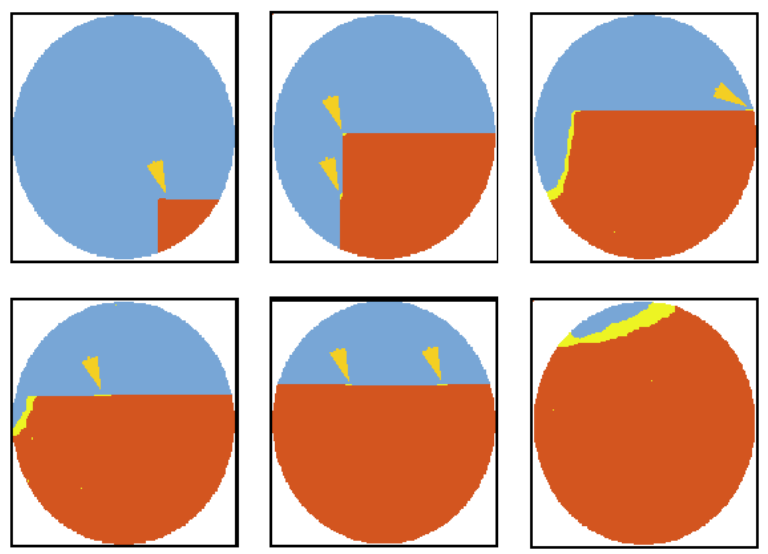

FIG. 15 (Color) Typical time evolution of a circular model particle of radius $r=120$ as it decays from the metastable state. Time increases from left to right and from top to bottom. The metastable (stable) phase is the blue (red) region, and we represent the specific avalanches at each time as yellow regions. As discussed in the main text, all the large avalanches (the only ones that are visible here to the naked eye; the small others are indicated by arrows) occur at the curved parts of the interface. This simulation is for $T=0.11 T_{\text {ons }}, p=10^{-6}$ and $h=-0.1$.

is the consequence of a competition between metastability, thermalization and the nonequilibrium perturbation. As a matter of fact, as it will be discussed below, observing power laws requires both free borders and the nonequilibrium condition. That is, the distributions $P(\Delta)$ look approximately exponential if the system has periodic borders and/or one sets $p=0$ in (2). It is also obviously convenient, in order to have good statistics, a choice of the parameter values that selects a region of the phase diagram in which evolution proceeds by as many jumps as possible. In any case, however, the behavior that we describe here is robust and characterizes our model, and no fine-tuning of parameters is needed.

\section{B. Many Scales}

We next show that the observed power laws are in the model a superposition of many individual processes, each with a well-defined scale, and that these may be traced back to specific demagnetization events. Therefore, there is no need here to invoke for any critical point, which is the familiar origin for scale invariance in equilibrium.

Let us consider a relatively-large circular model particle. Inspection of configurations then clearly reveals that, at sufficiently low $T$, the interesting events always occur at the interface between the metastable and stable phases. One observes curved domain walls due to the faster growth of the stable phase near the concave open borders. This is in accordance with a previous conclusion that, in equilibrium, the critical cluster always nucleates at the free border (17; 18). Any curvature costs energy, so that the large avalanches tend to occur at curved domain walls, which will tend to produce flatter interfaces; see Fig. 15. This is confirmed when one monitors the mean avalanche size $\left\langle\Delta_{m}\right\rangle$ and the wall curvature $\langle\kappa\rangle$ as a function of magnetization $m(t)$. We define the curvature $\kappa$ as the number of ascending steps at the interface. That is, the number of up spins which have two ups and two downs at the respective sides along the interface. (This definition requires well-defined compact clusters, which are the rule at low T.) The left graph in Fig. [16 illustrates our results. After averaging over many runs, definite correlations show up. That is, as one could perhaps have imagined, the event typical size is determined by the curvature of the interface region at which the avalanche occurs.

This is also confirmed by monitoring $P\left(\Delta_{m} \mid \kappa\right)$, the conditional probability that an avalanche of size $\Delta_{m}$ develops at a wall of curvature $\kappa$. We studied this in detail by simulating an interface of constant curvature that evolves according to the transition rate (2); see appendix C. The right graph of Fig. 16 shows that $P\left(\Delta_{m} \mid \kappa\right)$ has two regions for given $\kappa$. The first one corresponds to the small events of typical size (28) mentioned above. There is also a region which, contrary to the situation in Fig. 14, exhibits (stretched-) exponential behavior, namely, $P\left(\Delta_{m} \mid \kappa\right) \sim \exp \left[-\left(\Delta_{m} / \bar{\Delta}_{m}\right)^{\eta}\right]$ with $\eta \approx 0.89$. That is, a region of the interface with curvature $\kappa$ tends to induce avalanches of typical size $\bar{\Delta}_{m}(\kappa)$.

This fact turns out most relevant because, due to competition between the randomness induced by free borders and the one induced by $p$ in the transition rate (2), the interface tends to exhibit a broad range of curvatures with time, as illustrated in Fig. 15 and the left graph in Fig. 16. More specifically, relaxation proceeds via a series of 

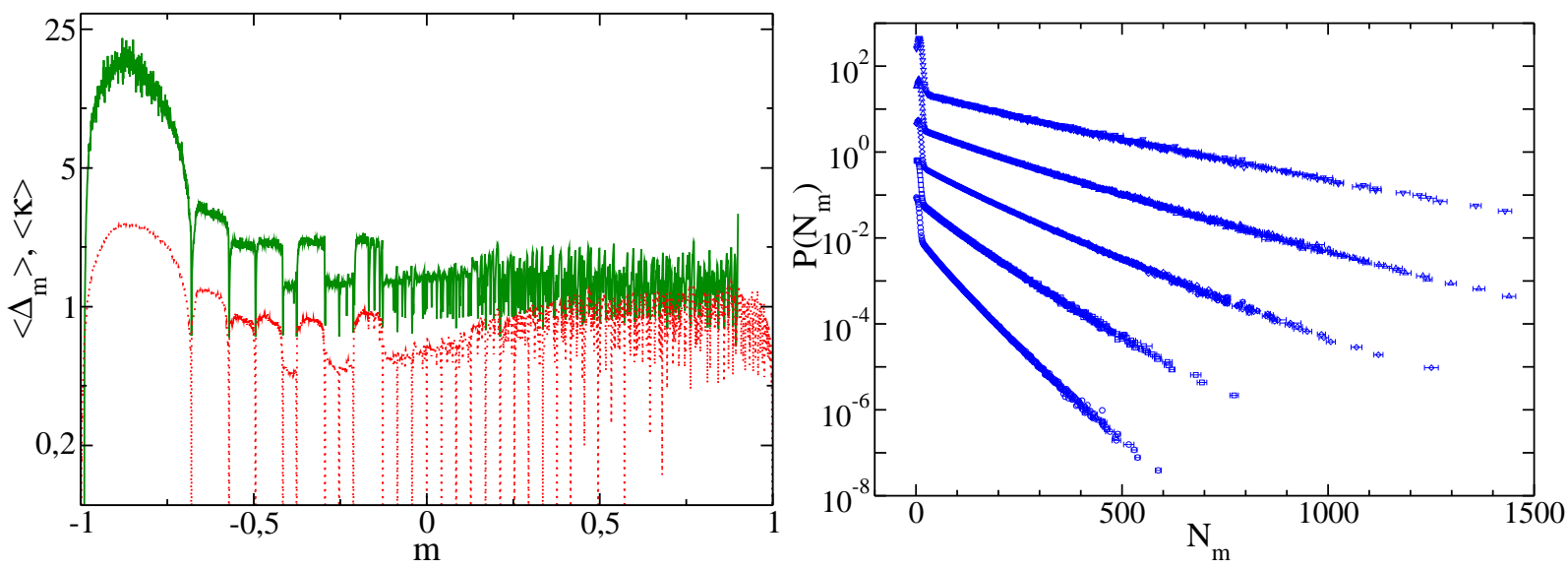

FIG. 16 (Color) Left: Semilogarithmic plot of the mean size $\left\langle\Delta_{m}\right\rangle$ (solid, green line) and mean curvature $\langle\kappa\rangle$ (dotted, red line) as a function of magnetization. Notice the non-trivial structure uncovering a high degree of correlation between the avalanche mean size and the average curvature of the wall at which it originates. Right: Semilogarithmic plot of $P\left(\Delta_{m} \mid \kappa\right)$, the size distribution for avalanches developing at a wall of constant curvature, $\kappa$, where $\kappa$ increases from bottom to top (see appendix C). Here, $N_{m} \equiv \frac{1}{2} N \Delta_{m}$. For visual convenience, the curves are shifted vertically by $10^{n}$ with $n=0$ to 4 from bottom to top. This corresponds to an average over 3500 independent runs, and running averages have also been performed for clarity purposes.

different configurations, each characterized by a typical curvature of the interface and by the consequent typical form of the critical cluster which induces the avalanche. Therefore, what one really observes when averaging over time is a random combination of many different avalanches, each with its typical well-defined (gap-separated) size and duration, which results in an effective distribution. The fact that this combination depicts several decades (more the larger the system is) of power-law behavior can be understood on simple grounds.

Let $Q(A)$ the probability of $A$, and $P(x \mid A)=A \exp (-A x)$ the probability of an event of size $x$ given $A$. Assume that $A$ can take a finite number of equally spaced values $A_{k}, k=0,1,2, \ldots, n$, in the interval $\left[A_{\min }, A_{\max }\right]$, so that $A_{k}=A_{\min }+k \delta$ with $\delta=\left(A_{\max }-A_{\min }\right) / n$ (alternatively, one may assume randomly distributed $A_{k} \mathrm{~s}$ ), and that all of them have the same probability, $Q(A)=$ const. One obtains that

$$
P(x)=\frac{\delta \mathrm{e}^{-x A_{\min }}}{1-\mathrm{e}^{-x \delta}}\left[A_{\min }-A_{\max } \mathrm{e}^{-(n+1) x \delta}-\delta \frac{1-\mathrm{e}^{-n x \delta}}{1-\mathrm{e}^{x \delta}}\right] .
$$

The fact that even such a simple, uncorrelated ansatz describes qualitatively the data is illustrated in Fig. 17. That is, the superposition of a large but finite number of exponential distributions, each with a typical scale, yields an effective global distribution which is consistent with apparent scale invariance. This distribution extends in practice up to a cutoff, $\exp \left(-x A_{\min }\right)$, which corresponds to the slowest exponential relaxation. Notice that eq. (34) predicts a size-independent exponent, $\tau(r)=\tau_{\infty}=2$, that differs somewhat from the observed asymptotic one, $\tau_{\infty}=1.71(4)$. This reveals that a more complete explanation than (34) would be suitable. This would require taking into account, for example, dynamic correlations such as the ones revealed in the left graph of Fig. 16. The above, however, is already semi-quantitative and there is no evidence that a more involved computation would modify qualitatively this picture.

Consider next $P\left(\Delta_{t} \mid \Delta_{m}\right)$, i.e., the probability that the avalanche of size $\Delta_{m}$ lasts a time $\Delta_{t}$. As expected from the above, this exhibits well-defined peaks corresponding to large correlations, i.e., avalanches of a given size have a preferred duration and vice versa. Assuming $\Delta_{m} \sim \Delta_{t}^{\gamma}$, we obtain $\gamma=\beta_{m} / \beta_{t}=1.52(5)$. Using this relation, one may obtain the duration distribution by combining eq. (34) with $P\left(\Delta_{m}\right) \mathrm{d} \Delta_{m}=P\left(\Delta_{t}\right) \mathrm{d} \Delta_{t}$. A comparison of the resulting curve with data in Fig. 17 leads to $\gamma \simeq 1.52$, in perfect agreement with the value obtained from the cutoff exponents $\beta$. More generally, a scaling plot of $P\left(\Delta_{t}\right)$ vs. $c \Delta_{t}^{\gamma}$, with $c$ some proportionality constant, must collapse onto the corresponding curve $P\left(\Delta_{m}\right)$ for each particle radius $r$. This is confirmed in the left graph of Fig. 14 for $\gamma \simeq 1.52$, further supporting our description of scales superposition. Let us assume for a moment that, as it would occur in the presence of a critical condition, both $P\left(\Delta_{m}\right)$ and $P\left(\Delta_{t}\right)$ are true (as opposed to apparent) power-law distributions. It then follows the scaling relation $(\alpha-1)=\gamma(\tau-1)$ which, together with our values above for $\alpha$ and $\tau$, implies that $\gamma \simeq 1.76$, which contradicts the value $\gamma \simeq 1.52$ that follows by two methods. We believe this misfit simply confirms our point that none of the distributions $P(\Delta)$ in this section exhibits true scaling behavior. 

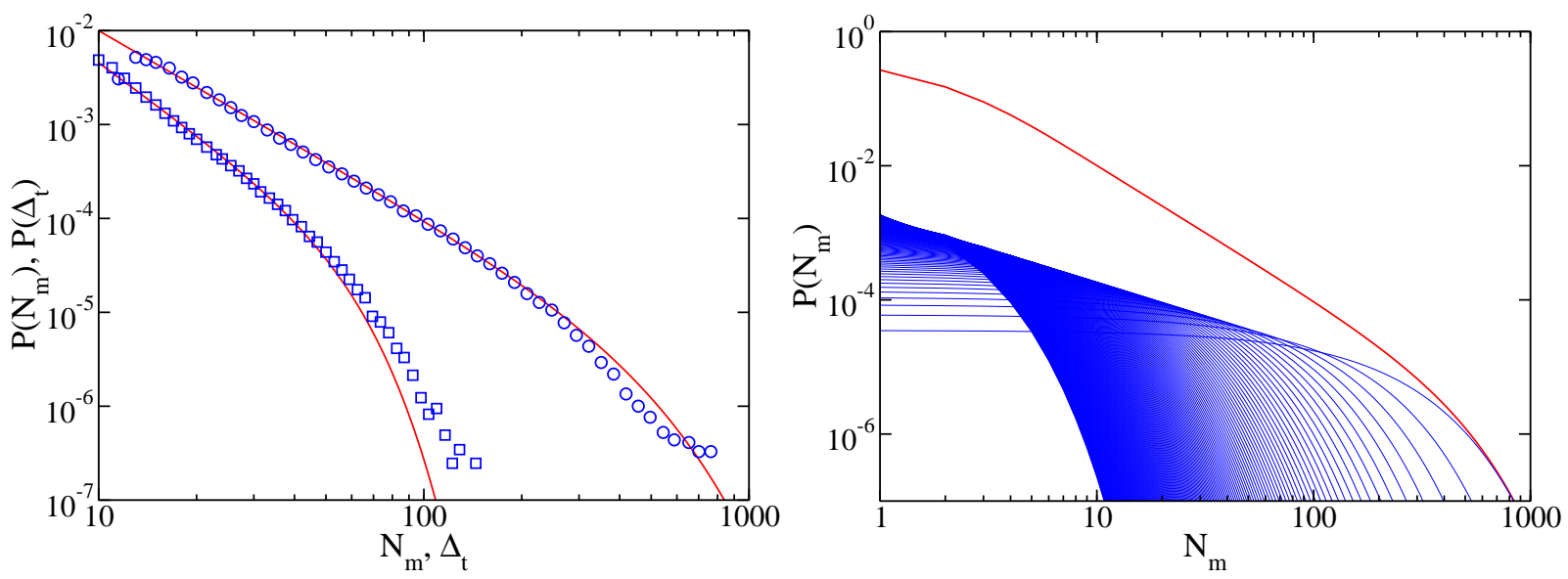

FIG. 17 (Color) Left: Solid lines are the predictions, equation (34) for $n=200, A_{\min }=0.007$ and $A_{\max }=1$. The symbols stand for the avalanches duration (lower curve) and size (upper curve) when $r=60$, i.e., two of the data sets in figures 2 and 3. In this particular case, the finite-size exponent is $\tau(r=60)=2.06(2)$. Right: The theoretical avalanche size distribution on the left panel, and the many exponentials which compose it.

\section{Some Additional Comments}

We conclude that the model particle relaxes via events, each with a well-defined scale, but many of them randomly combine producing (apparent) power-law behavior. This is a consequence in the model of two main features, namely, free borders and the nonequilibrium perturbation. (For $p=0$ and/or periodic boundary conditions, one just observes a well-defined mean.) There is no indication of chaos, e.g., sensitivity to the initial condition. We also discard that the observed power laws have any relation in the model with criticality in the familiar, equilibrium sense. In fact, we do not observe any relevant correlation other than the dynamic ones that we described in detail. The question, given that our model is purposely oversimplified, is whether this picture applies to scale-free fluctuations in natural phenomena. Demonstrating this, i.e., analysis of separate elementary events in actual cases, is difficult. However, we argue next that there are some indications that this may be the case in some occasions.

We first mention that our separation between small events — as described in eq. (28) and appendix B - and (large) avalanches is also supported by experiments $(35 ; 36 ; 37)$. On the other hand, it is remarkable that the statistical properties of the resulting distributions are indistinguishable in practice from actual data (38). For instance, size corrections similar to the ones in eqs. (30) and (32) for $\tau$ and $\alpha$, respectively, have been reported in avalanche experiments on rice piles (39), and our asymptotic values are very close to the ones reported in magnetic experiments, e.g., $\tau_{\infty}=1.77(9), \alpha_{\infty}=2.22(8)$ and $\gamma=1.51(1)$ in Ref. (35) for (quasi-two dimensional) ribbons; see also Ref. (37). Moreover, our cutoff values in eq. (33) follow the precise trend observed, for instance, in magnetic materials (40; 41). Our non-critical scenario is also consistent with the fact that one variously observes either power-law or exponential distributions, or a mixture of both, in different but closely related experiments and even in different regimes of the same experiment; see, for instance, Ref. (42).

It is also remarkable that our picture does not imply universality; instead, microscopic details matter, as it is the case in experiments. That is, universality is suspicious in a context in which critical exponents seem to vary with conditions and materials $(35 ; 41)$, and applications sometimes rely on sensibility to the sample microstructural details (43; 44). Our picture also explains other features of natural signals such as, for instance, reproducibility. This refers to the fact that experiments reveal that the avalanches, i.e., excluding the small events, tend to occur at the same stages of evolution (45; 46; 47). As shown in Fig. 18, our system exhibits a high degree of reproducibility due to the strong tendency of the critical cluster to nucleate at the border. Simulations indicate that this occurs more markedly the lower $h$ and $T$ are, i.e., when the system is more efficient in selecting the most (energetically) favorable configuration.

Interesting enough, if our picture has a broad applicability, there would be more hope to the goal of predicting large events. That is, the assumption of some underlying criticality naturally implies that catastrophes, though relatively rare, occur in an strongly correlated bulk and, consequently, have the same cause as the smaller avalanches (48; ; 49; ;50). Instead, in our picture, events are characterized by their size, and each size follows from some specific microscopic configuration. The configurations that, under appropriate conditions, may originate large events qualitatively differ from the ones corresponding to smaller events. In summary, there is some specific cause for each event which depends on its size.

In spite of the extreme simplicity of the model, one should perhaps recognize that it is likely that its basic features 


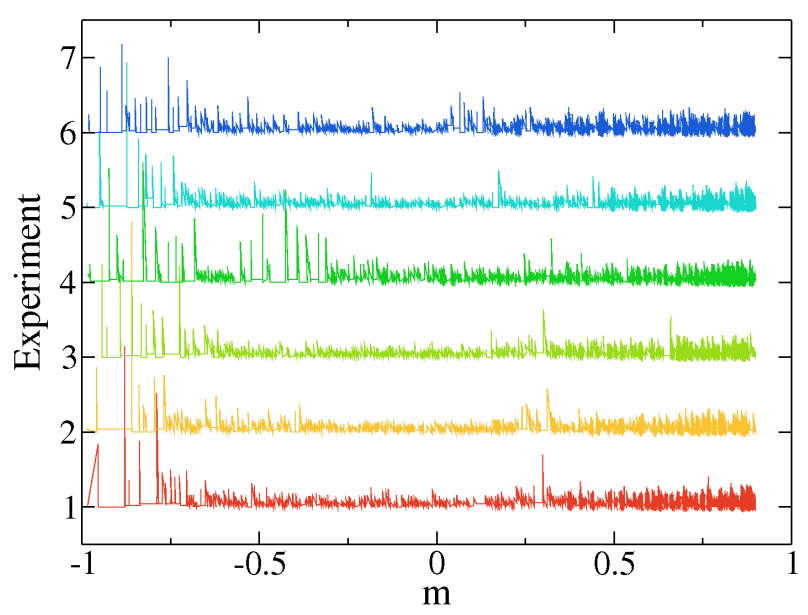

FIG. 18 (Color) The avalanche sizes as a function of magnetization for six typical independent runs. This is to illustrate that large avalanches tend to occur, approximately, at the same value of magnetization. This occurs here for the large avalanche around $m=0.3$ in four of these runs and for the sequence of large avalanches in all the runs for $m \in[-0.7,-1]$, for instance.

are present in some actual systems as well. In particular, one may imagine that the model randomness mimics the causes for the random interface rearrangements in magnets and for the slip complexity in earthquakes, for instance. Furthermore, in these two cases, as in the model, time evolution is through a series of successive short-lived states that constantly halt the system relaxation. It is precisely this what causes a constant change of scale which results in the (apparent) scale invariance of the model.

It would be interesting to study the possible occurrence of these short-lived halt states in natural signals. These halts are associated in the model particle with flat interface regions. That is, once the initial metastability breaks down, the particle becomes inhomogeneous, and flat interfaces have a significant probability to form after each avalanche (which aims at minimizing interfacial energy). As this is the most stable configuration against small perturbations, the system remains some time with constant magnetization $m\left(t_{b}\right)$. This may be described as an entropic metastability. There is no energy barrier but an unstable situation such that a given microscopic random event suffices to initiate the next avalanche.

Finally, we mention that there are many other possible explanations for scale-invariant noise based on non-critical mechanisms; see, for example, Refs. $(45 ; 51 ; 52 ; 53 ; 54 ; 555 ; 56)$. To our knowledge, however, this is the most general one so far reported which has a physics content. Other proposal are often restricted to specific situations and, in some cases, they may also be interpreted at the light of a superposition of many different typical scales. A similar origin for electronic $1 / f$ noises was suggested in the past (see, for instance, Refs. $(45 ;$; $5 ;$; $58 ; 59 ; 60)$ ), though this is perhaps the first time in which an explicit relation is drawn between elementary events (avalanches) and microscopic physical processes. We also mention that recent observations in a sociological context may be cast into the behavior of our "particle". That is, according to certain definitions, there is a relation between fame and merit (61). It has recently been estimated that the probability distribution for a certain level of fame falls off exponentially for a respected scientist, while it seems to decay as a power law in other social groups (62) This may reflect that fame in science is mainly due to a homogeneous, rather well-informed community, while many different communities, each with a different perception of merit contribute to the fame of, for instance, a movies actor. The combination of distributions with different means then produces the observed power law.

\section{CONCLUSION}

This paper deals with metastability in a nonequilibrium environment. We studied a two-dimensional Ising ferromagnet subject to competing dynamics, as if a completely random processes were constantly perturbing a tendency to thermalization at temperature $T$. The perturbation impedes equilibrium, even if it is extremely weak, and a nonequilibrium steady state sets in asymptotically. This is a convenient background to investigate various questions, and it could mimic certain nonequilibrium situations in nature.

In the light of the observed qualitative behavior, we argue that, at a mesoscopic level, concerning both steady and time-dependent properties of clusters in metastable conditions, it is sensible to assume that a nonequilibrium free-energy function exists which is formally similar to the equilibrium concept. In particular, we assume that the metastable phase results from a contest between surface and bulk terms of this function, and that a nonequilibrium 
surface tension captures the (strongly nonlinear) interplay between thermal and nonequilibrium noises. We also show that a Langevin equation with additive and multiplicative noises captures the essential physics.

Our theoretical approach predicts resonant coupling among the thermal and nonequilibrium noises which results in novel phenomenology. For instance, there is noise-enhanced propagation of domain walls and stabilization of the metastable state at low $T$, and reentrant behavior of the spinodal field under strong nonequilibrium conditions. These cooperative phenomena are perfectly confirmed by Monte Carlo simulations.

We also explored the relaxation from a metastable state in the presence of open boundaries. This case also shows intriguing behavior at low $T$. That is, the decay occurs through a series of many metastable-like states that repeatedly halt the dynamics, which resembles the relaxation by avalanches reported for many complex systems, e.g., interface rearrangements in magnets and fault slips causing earthquakes. We conclude that scale-free avalanche distributions are not to be associated to any critical condition in the model but are simply a superposition of many different classes of events, each with a well-defined scale. In the model this is determined by the curvature of the domain wall at which the avalanche originates. This may be a property of any complex relaxation phenomena characterized by a multiplicity of short-lived, metastable-like states.

\section{Acknowledgments}

We acknowledge useful discussions with Miguel Ángel Muñoz, and financial support from Ministerio de Educación y Ciencia, FEDER Funds, European Union, and Junta de Andalucía, through projects FIS2005-00791, HPRN-CT2002-00307 (DYGLAGEMEM) and FQM-165.

\section{Appendix A: The Spinodal Field in MC Simulations}

We briefly describe here a method to estimate the spinodal field $h^{*}(T, p)$ which separates metastable from unstable states. To be precise, $h^{*}$ is a pseudo-spinodal given that the transition in the actual system (with fluctuations) is not sharp but a progressive crossover (34), as illustrated in the inset to right graph in Fig. 12, Estimating $h^{*}$ allows in the main text a confirmation of the corresponding mean-field prediction.

Our method consists in following the system path in phase space as it evolves from the initial state with all spins up toward the final stable state. With this aim, we assign to each intermediate state along this path, say $\mathbf{s}_{k}$, a measure $\Lambda\left(\mathbf{s}_{k}\right)$ of the net tendency of the system to evolve toward the stable phase from that configuration. Using the concept of spin class described in $₫$ III.A , it is straightforward to see that $\Lambda\left(\mathbf{s}_{k}\right)=\mathcal{G}\left(\mathbf{s}_{k}\right)-\mathcal{S}\left(\mathbf{s}_{k}\right)$, where

$$
\mathcal{G}\left(\mathbf{s}_{k}\right) \equiv \sum_{n=0}^{4} \nu\left(+, n ; \mathbf{s}_{k}\right) \omega(+, n) ; \quad \mathcal{S}\left(\mathbf{s}_{k}\right) \equiv \sum_{n=0}^{4} \nu\left(-, n ; \mathbf{s}_{k}\right) \omega(-, n)
$$

are the growth and shrink rates of the stable phase in state $\mathbf{s}_{k}$, respectively (recall that $\left.h<0\right)$. Here, $\nu\left(s, n ; \mathbf{s}_{k}\right)$ is the fraction of spins in class $(s, n)$ for configuration $\mathbf{s}_{k}$, and $\omega(s, n)$ is the spin-class transition rate.

Metastability is hampered by free-energy barriers, while unstable states evolve without any impediment. Therefore, we may divide relaxation paths in phase space in two sets: Metastable paths, for which at least one configuration exists such that $\Lambda\left(\mathbf{s}_{k}\right)<0$, and unstable paths, where $\Lambda\left(\mathbf{s}_{k}\right)>0 \forall k$. Given the stochasticity of the dynamics, one needs to be concerned with the probability of occurrence of metastability, defined as $\Pi(T, p, h)=n_{\text {met }} / N_{\text {exp }}$, where $n_{\text {met }}(T, p, h)$ is the number of experiments out of the total $N_{\exp }$ in which the relaxation path in phase space belongs to the class of metastable paths. The limit of metastability is defined in this scheme as the field for which $\Pi\left(T, p, h^{*}\right)=0.5$. This is illustrated in the right graph of Fig. 12 for a system size $L=53$. (We studied finite-size corrections to the spinodal field by simulating larger systems; however, these corrections may be neglected for all practical purposes.) This confirms the reentrant behavior of $h^{*}$ for low temperature under strong nonequilibrium conditions.

\section{Appendix B: Small Events}

We now determine the avalanche statistics for a flat interface under a magnetic field at low $T$. Let us assume a macroscopic, perfectly flat interface between the metastable and stable phases which, for $h<0$, correspond to the up and down phases, respectively.

Flat interfaces are very likely at low $T$, since they minimize surface tension, and they will tend to invade the metastable phase with time for any $h<0$.

We are interested in the probability per unit time for a change in the state of a spin in class $(s, n), W(s, n)$, where $s= \pm 1$ and $n \in[0,4]$ stands for the spin number of up NNs; see $₫$ III.A One has that $W(s, n)=\nu(s, n) \omega(s, n)$, where 

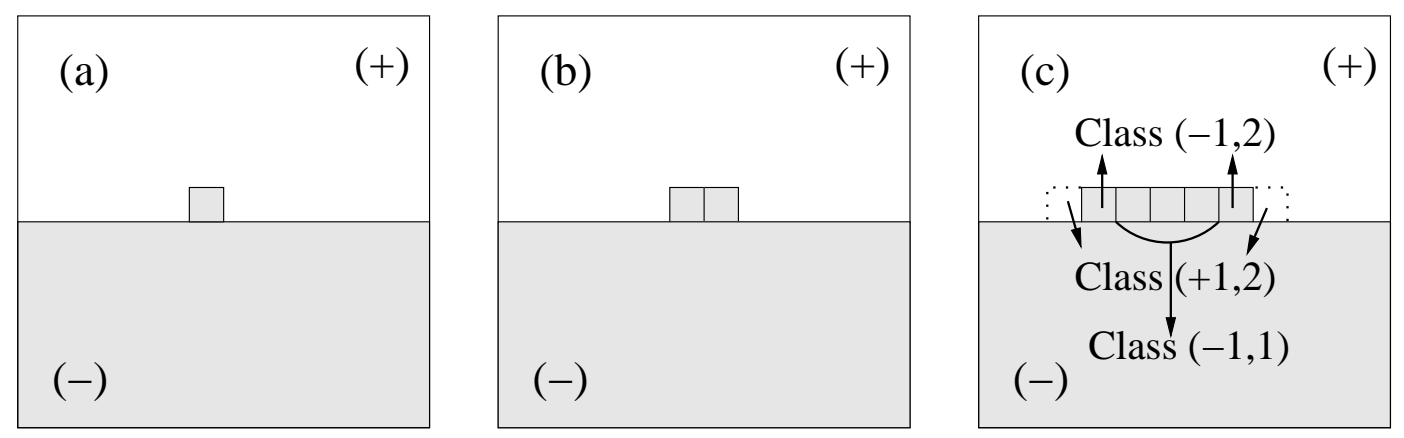

FIG. 19 Schematic representation of a flat interface in which a nucleation event originates and grows as discussed in the main text. Notice in (c) that the only relevant spins during this kind of growth are the two spins in class $(-1,2)$ and the two spins in class $(+1,2)$ at the respective ends of the cluster.

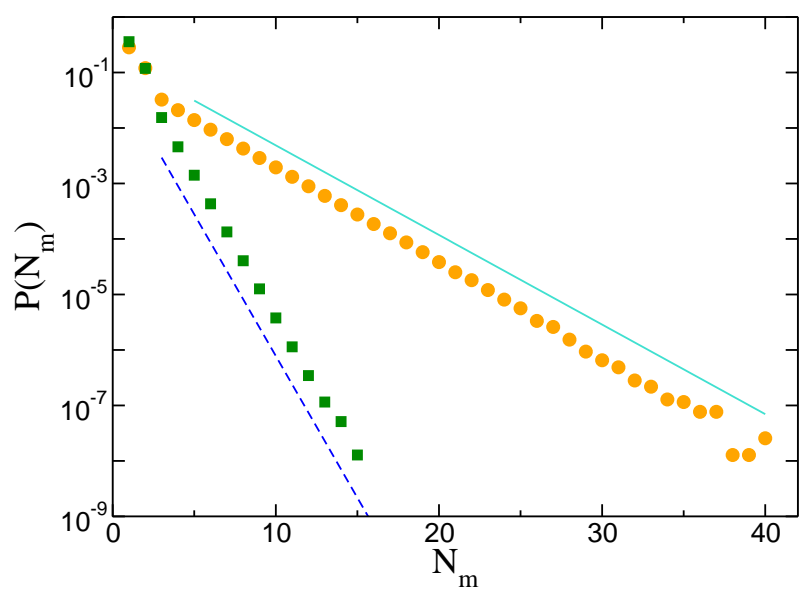

FIG. 20 Semilog plot of the avalanche size distributions $P\left(N_{m}\right)$ for avalanches in the field direction $(\bigcirc)$ and against the field $(\square)$, as obtained from Monte Carlo simulations of a flat domain wall of size $L=53$ for $T=0.11 T_{\text {ons }}, p=10^{-6}$ and $h=-0.1$. The slope of the lines correspond to our theoretical predictions in eqs. (35) and (36).

$\nu(s, n)$ is the fraction of spins of class $(s, n)$ in the current configuration and $\omega(s, n)$ is the corresponding transition rate; see eq. (2) and Table [. For the given initial condition, the only possible classes are $(+1,4),(+1,3),(-1,1)$ and $(-1,0)$. For $0 \leq|h| \leq 2 J$ and low $T, \omega(s, n)$ is very small for these four classes, e.g., between $10^{-6}$ and $1.24 \times 10^{-6}$ for $T=0.11 T_{\mathrm{ons}}, p=10^{-6}$ and $h=-0.1$.

Let us assume that a fluctuation nucleates as in Fig. 19a, i.e., one spin has been flipped. This spin belongs to class $(-1,3)$, and its two NN spins in the direction of the interface belong to class $(+1,2)$. It is straightforward to show that the probability that this fluctuation either grows along the interface or shrinks is much larger than the probability of any other event in the surrounding bulk.

Given that there are many more spins in the bulk than in a flat interface, there will be many bulk fluctuations before anything happens at the interface. However, whenever an interface fluctuation occurs, an avalanche will quickly develop along the wall before anything disturbs the surrounding bulk.

Therefore we can safely assume that once the interfacial perturbation has appeared, the system dynamics can be reduced to the growth and shrinkage dynamics of the interfacial perturbation. Under this assumption, the most probable process to be observed consists in the growth of the interfacial fluctuation via the flipping of the lateral spins in class $(+1,2)$ at the extremes, until one of the two spins in class $(-1,2)$ which bound the interfacial fluctuation flips, halting the avalanche (see Fig. 19, c).

We now assume that the system is in the state depicted in Fig. 19, b, with two up interfacial spins flipped, and we want to compute the probability of finding a lateral avalanche of size $N_{m}$, i.e. involving $N_{m}$ spins. The restricted dynamics we assume only involves four different spins [two in class $(+1,2)$ and two in class $(-1,2)$ ]. Within our 


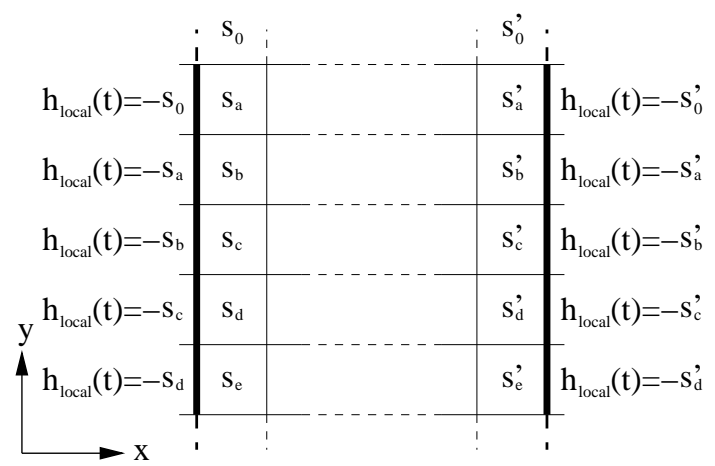

FIG. 21 Sketch of the dynamic boundary conditions in the $\hat{x}$-direction. Here we represent the first and last columns of the system, and the local, dynamic magnetic field that each spin in these columns feels as the system evolves in time. The effect of these dynamic boundary conditions is equivalent to eliminate the interaction of each border spin with its up neighbor, i.e. concave open boundary conditions.

constrained dynamics, the probabilities for the fluctuation to grow or stop are

$$
\begin{aligned}
\chi_{\text {grow }} & =\frac{1}{1+p}\left[p+(1-p) \frac{\mathrm{e}^{2 \beta|h|}}{1+\mathrm{e}^{2 \beta|h|}}\right], \\
\chi_{\text {stop }} & =\frac{1}{1+p}\left[p+(1-p) \frac{\mathrm{e}^{-2 \beta|h|}}{1+\mathrm{e}^{-2 \beta|h|}}\right] .
\end{aligned}
$$

The probability of finding a lateral avalanche of size $N_{m}$ is hence $P\left(N_{m}\right)=\chi_{\text {grow }}^{N_{m}} \chi_{\text {stop }} \equiv \chi_{\text {stop }} \exp \left(-N_{m} / \bar{\Delta}_{m}^{(-)}\right)$, which defines $\bar{\Delta}_{m}^{(-)}$, the typical size characterizing avalanches in the field direction

$$
\bar{\Delta}_{m}^{(-)}=\frac{1}{\ln \left[\frac{(1+p)\left(1+\mathrm{e}^{2 \beta|h|}\right)}{p+\mathrm{e}^{2 \beta|h|}}\right]} .
$$

In a similar way we can compute the typical size $\bar{\Delta}_{m}^{(+)}$of avalanches against the magnetic field, i.e. avalanches involving spins in the stable (down) phase. The result is

$$
\bar{\Delta}_{m}^{(+)}=\frac{1}{\ln \left[\frac{(1+p)\left(1+\mathrm{e}^{-2 \beta|h|}\right)}{p+\mathrm{e}^{-2 \beta|h|}}\right]} .
$$

As expected, $\bar{\Delta}_{m}^{(+)}<\bar{\Delta}_{m}^{(-)}$. Fig. 20 shows the size probability distributions $P\left(N_{m}\right)$ for avalanches toward and against the field, as obtained in Monte Carlo simulations of a flat domain wall of size $L=53$, for for $T=0.11 T_{\text {ons }}, p=10^{-6}$ and $h=-0.1$. A comparison with our predictions (35) and (36) is also shown, with excellent results.

The small avalanches here described, to be associated with the presence of flat domain walls as the system demagnetizes from the initial metastable state, appear in our magnetic nanoparticle together with more structured events of large size. As shown in section IV these structured events exhibit scale-invariant properties which stem from the interplay between the nonequilibrium perturbation and the free borders of the magnetic particle. A good measurement of these large-scale avalanches involves filtering the above trivial noise, also known as extrinsic noise (35).

\section{Appendix C: Avalanches from Walls of Constant Curvature}

We have shown in $\oint$ IV.B that large avalanches originate due to domain wall curvature ${ }^{5}$. Moreover, domain wall curvature appear in the system due to concave open borders, since spins near the concave border flip faster than bulk spins.

\footnotetext{
${ }^{5}$ In this context, we define the amount of curvature of a domain wall as the number of kinks in the interface, e.g., the number of up spins flanked, respectively, by two ups and by two downs at the sides along the interface). This definition requires well-defined compact clusters, as for low temperature.
} 

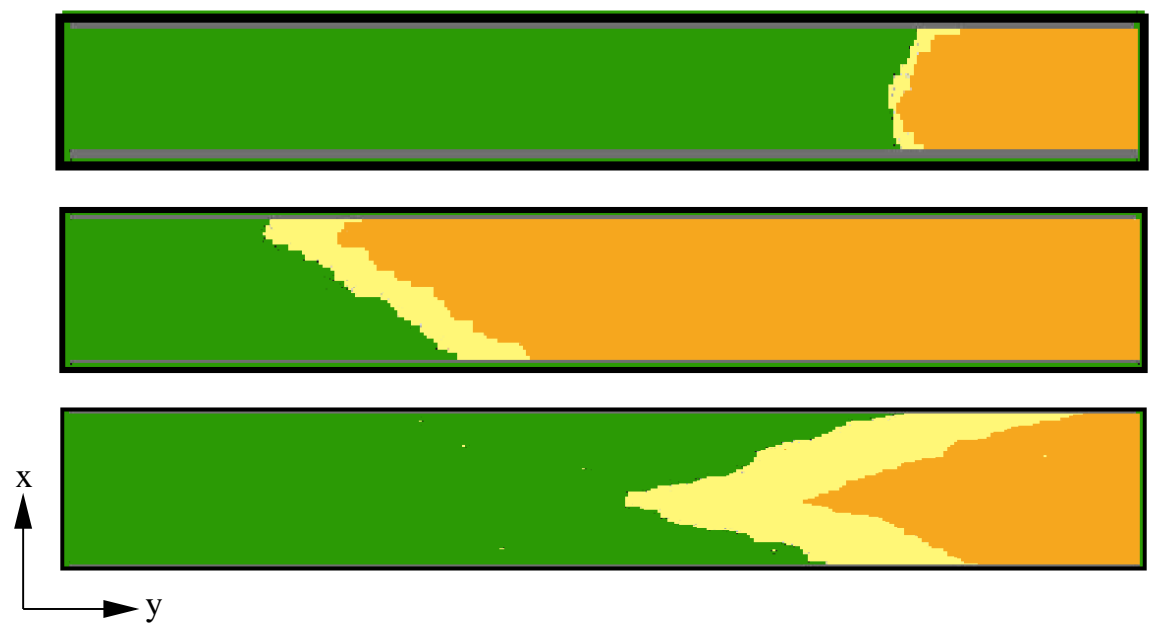

FIG. 22 Snapshots of an interface that evolves according to transition rate (2) subject to concave open boundaries as described in the text. The stable (metastable) phase corresponds to the green (orange) regions. The yellow regions correspond to the avalanches in light yellow. From top to bottom, $L_{\mathrm{x}}=20,40$, and 100 .

The MC simulations reported in $₫$ IV.B show that the size of the avalanche is strongly correlated with the curvature of the interface region at which the event originates. Here we deep on this correlation, namely, the interest is on the probability that an avalanche of size $N_{m}$ originates at a wall of some specific curvature. With this aim, we modified our basic system so that it shows an interface with constant, except for small fluctuations, non-zero curvature.

Consider a semi-infinite, $L_{\mathrm{x}} \times \infty$ lattice with concave open boundary conditions in the $\hat{x}$ direction. This is done in practice starting with a $L_{\mathrm{x}} \times L_{\mathrm{y}}$ lattice and fixing the spins at the top row to +1 , while spins in the lowest row are fixed to -1 . On the other hand, boundary conditions in the $\hat{x}$ direction are dynamic: the lattice is also open in the $\hat{x}$ direction, although each spin in the first and last column suffers an additional dynamic magnetic field, equal at any time to the negative value of its up neighbor. This is sketched in Fig. 21, For each spin in the first and the last columns, the effect of these dynamic boundary conditions is to effectively decouple this spin from its up neighbor. In this way we mimic a concave, stair-like border (as the one found by the domain wall in the circular nanoparticle at some stages of its evolution, see Fig. 15), with a fixed distance $L_{\mathrm{x}}$ between both concave borders.

We initialize the system with a flat domain wall between the metastable and stable phases. Under the action of the magnetic field, the domain wall propagates toward the metastable phase. Due to the concave boundary conditions in the $\hat{x}$ direction, the interface propagates faster near the boundary. After a short transient, the initially flat domain wall reaches an stationary state, with an almost constant (up to small fluctuations) non-zero curvature, which depends linearly on the size $L_{\mathrm{x}}$, see Fig. 22. In order to emulate an infinite system in the $\hat{y}$ direction, we shift the observation window whenever the domain wall gets close to the system extreme in the propagation direction, always keeping the interface inside the system. In practice we generate a new region with up (metastable) spins in one extreme of the system whenever the above condition is met, eliminating an equivalent region of down (stable) spins in the opposite extreme. (In fact, in order to give time for the newly introduced spin region to relax to the typical state of the metastable phase at the given $T, p$ and $h$, we perform the shift well in advance before the domain wall reaches the boundary.).

The above defined system evolves via avalanches, whose distribution we can measure. In this way we obtain a reliable measure of avalanche statistics for a domain wall with (approximately) constant curvature, see the right graph in Fig. 16. In particular, we observe that domain walls with constant curvature exhibit avalanches with a well-defined typical scale.

\section{References}

[1] J.S. Langer, Ann. Phys. 41, 108 (1967); 54, 258 (1969).

[2] J.L. Lebowitz, O. Penrose, J. Stat. Phys. 3, 211 (1971).

[3] O. Penrose, J.L. Lebowitz, Towards a Rigorous Molecular Theory of Metastability, in Fluctuation Phenomena, E. Montroll, J.L. Lebowitz (eds.), North-Holland, Amsterdam (1979) — there is a 2nd edition dated 1987.

[4] J.D. Gunton, M. Droz, Introduction to the Theory of Metastable and Unstable States, Springer-Verlag, Berlin (1983). 
[5] J.S. Langer, An Introduction to the Kinetics of First-Order Phase Transitions, in Solids Far from Equilibrium, C. Godrèche (ed.), Cambridge University Press, Cambridge (1992).

[6] P.A. Rikvold, H. Tomita, S. Miyashita, S.W. Sides, Phys. Rev. E 49, 5080 (1994).

[7] P.G. Debenedetti, Metastable Liquids. Concepts and Principles, Princeton University Press, Princeton (1996).

[8] G. Báez, H. Larralde, F. Leyvraz, R. A. Méndez-Sánchez, Phys. Rev. Lett. 90, 135701 (2005).

[9] S. Franz, Europhys. Lett. 73, 492 (2006).

[10] P.G. Debenedetti, F.H. Stillinger, Nature 410, 267 (2001).

[11] J. Marro, R. Dickman, Nonequilibrium Phase Transitions in Lattice Models, Cambridge University Press, Cambridge (1999) - there is a paperback edition dated 2005.

[12] E.J. Neves and R.H. Schonmann, Comm. Math. Phys. 137, 209 (1991); Prob. Theor. Rel. Fields 91, 331 (1992).

[13] R.H. Schonmann, Comm. Math. Phys. 147, 231 (1992); 161, 1 (1994).

[14] C.C.A. Gunter, P.A. Rikvold and M.A. Novotny, Phys. Rev. Lett. 71, 3898 (1993); Physica A 212, 194 (1994).

[15] P.A. Serena and N. García, in Quantum Tunneling of Magnetization, L. Gunther and B. Barbara, eds., Kluwer, Dordrecht 1995.

[16] J. Marro and J.A. Vacas, Phys. Rev. B 56, 1 (1997).

[17] H.L. Richards, M. Kolesik, P.A. Lindgard, P.A. Rikvold, M.A. Novotny, Phys. Rev. B. 55, (1997) 11521.

[18] E.N.M. Cirillo and J.L. Lebowitz, J. Stat. Phys. 90, 211 (1998).

[19] P.L. Garrido, J. Marro. Phys. Rev. Lett. 621929 (1989).

[20] R.A. Blythe, M.R. Evans, Phys. Rev. Lett. 89, 080601 (2002).

[21] S.M. Dammer, S.R. Dahmen, H. Hinrichsen, J. Phys. A 35, 4527 (2002).

[22] P.I. Hurtado, J. Marro, P.L. Garrido, Phys. Rev. E 70, 021101 (2004).

[23] R. Dickman, Phys. Lett. A 122, 463 (1987).

[24] R. Kikuchi, Phys. Rev. 81, 988 (1951).

[25] P.I. Hurtado, P.L. Garrido, J. Marro, Phys. Rev. B 70, 245409 (2004).

[26] W.K. Burton, N. Cabrera, F.C. Frank, Phil. Trans. Roy. Soc. (London), Ser. A 243, 299 (1951).

[27] J.E. Avron, H. van Beijeren, L.S. Schulman, R.K.P. Zia, J. Phys. A 15, L81 (1982).

[28] E. Müller-Hartmann, J. Zittartz, Z. Phys. B 27, 261 (1977).

[29] G. Wulff, Kristallogr. Mineral. 34, 448 (1901); see also C. Herring, Phys. Rev. 82, 87 (1951).

[30] W.P. Wolf, Braz. J. Phys. 30, 794 (2000).

[31] M. A. Novotny, Phys. Rev. Lett. 74, 1 (1995); 75, 1424 (1995); M. Kolesik, M. A. Novotny, P. A. Rikvold, Phys. Rev. Lett. 80, 3384 (1998).

[32] R. Mantegna, B. Spagnolo, Phys. Rev. Lett. 75, 563 (1996).

[33] N.G. van Kampen, Stochastic Processes in Physics and Chemistry, North Holland (1981).

[34] K. Binder, H. Müller-Krumbhaar, Phys. Rev. B 9, 2328 (1974); D.W. Herrmann, W. Klein, D. Stauffer, Phys. Rev. Lett. 49, 1262 (1982); C. Unger, W. Klein, Phys. Rev. B 29, 2698 (1984).

[35] D. Spasojević, S. Burkvić, S. Milošević, H.E. Stanley, Phys. Rev. E 54, 2531 (1996).

[36] M. De Menech, A.L. Stella, C. Tebaldi, Phys. Rev. E 58, R2677 (1998); C. Tebaldi, M. De Menech, A.L. Stella, Phys. Rev. Lett. 83, 3952 (1999); M. De Menech, A.L. Stella, Phys. Rev. E 62, R4528 (2000).

[37] G.-P. Zheng, M. Li, J. Zhang, J. Appl. Phys. 92, 883 (2002).

[38] P.I. Hurtado, J. Marro, and P.L. Garrido, J. Stat. Mech. (2006) P02004.

[39] V. Frette, K. Christensen, A. Malthe-Sorenssen, J. Feder, T. Jossand, P. Meakin, Nature 379, 49 (1996).

[40] M. Bahiana, B. Koiller, S.L.A. de Queiroz, J.C. Denardin, and R.L. Sommer, Phys. Rev. E 59, 3884 (1999).

[41] G. Durin, S. Zapperi, Phys. Rev. Lett. 84, 4705 (2000).

[42] H.J. Jensen, Self-Organized Criticality, Cambridge University Press, Cambridge (1998).

[43] K.B. Klaassen and J.C.L. Vanpeppen, IEEE Trans. Magn. 26, 1697 (1990).

[44] L.B. Sipahi, J. Appl. Phys. 75, 6978 (1994); R.J. López, Rev. Sci. Instrum. 70, 171 (1999).

[45] M.B. Weissman, Rev. Mod. Phys. 60, 537 (1988); ibid, 65, 829 (1993).

[46] J.S. Urbach, R.C. Madison, J.T. Markert, Phys. Rev. Lett 75, 4694 (1995).

[47] S.M. Bezrukov and M. Winterhalter, Phys. Rev. Lett. 85, 202 (2000).

[48] P. Bak, How Nature Works, Copernicus, N.Y. (1996).

[49] R.N. Mantegna, H.E. Stanley, An Introduction to Econophysics: Correlations and Complexity in Finance, Cambridge University Press, Cambridge (2000).

[50] D. Sornette, Critical Phenomena in Natural Sciences, Springer-Verlag, Heidelberg (2000).

[51] E. Milotti, Phys. Rev. E 51, 3087 (1995).

[52] A. Bunde et al, Phys. Rev. Lett. 78, 3338 (1997).

[53] P. De Los Rios, Y.-C. Zhang, Phys. Rev. Lett. 82, 472 (1999).

[54] B. Kaulakys, Microelectr. Reliab. 40, 1787 (2000); also as ArXiv cond-mat/0305067.

[55] J. Davidsen, H.G. Schuster Phys. Rev. E 62, 6111 (2000); 65, 26120 (2002).

[56] M. E. J. Newman, Contemporary Physics 46, 323 (2005).

[57] J. Bernamont, Ann. Phys. (Leipzig) 7, 71 (1937).

[58] H. Sawada, J. Phys. Soc. Japan 7, 575 (1952).

[59] P. Mazzetti, Il Nuovo Cim. 25, 1322 (1962); 31, 88 (1964).

[60] I. Procaccia, H. Schuster, Phys. Rev. A 28, R1210 (1983).

[61] J.P. Bagrow, H.D. Rozenfeld, E.M. Bollt, and D. ben-Avraham, Europhys. Lett. 67, 511 (2004). 
[62] J.P. Bagrow and D. ben-Avraham, in Modeling Cooperative Behavior in the Social Sciences, AIP Conf. Proc. 779, pp. 81-90, American Institute of Physics, New York (2005). 